\title{
The slip velocity of nearly neutrally buoyant tracers for large-scale PIV
}

\author{
David Engler Faleiros $^{1,2}\left[\right.$ Marthijn Tuinstra ${ }^{1} \cdot$ Andrea Sciacchitano $^{2} \cdot$ Fulvio Scarano $^{2}$
}

Received: 18 August 2020 / Revised: 6 July 2021 / Accepted: 21 July 2021 / Published online: 19 August 2021

(c) The Author(s) 2021

\begin{abstract}
The behaviour of nearly neutrally buoyant tracers is studied by means of experiments with helium-filled soap bubbles and numerical simulations. The current models used for estimating the slip velocity of heavy micro particles and neutrally buoyant particles are reviewed and extended to include the effect of unsteady forces and particle Reynolds number. The particle motion is analysed via numerical simulations of a rectilinear oscillatory flow and in the flow around an airfoil within a particle flow parameter space that is typical of large-scale PIV experiments. An empirical relation is obtained that estimates the particle slip velocity, depending on the particle-to-fluid density ratio, the particle Reynolds number and frequency of the local flow fluctuations. The model developed is applied to assess the slip velocity of helium-filled soap bubbles in a largescale experiment conducted at the German-Dutch wind (DNW) tunnels in the flow around an airfoil, with chord Reynolds numbers up to three millions. Furthermore, a procedure is proposed that can be used to retrieve the bubbles mean density and dispersion from measurements of mean velocity and fluctuations, respectively.
\end{abstract}

\section{Graphic abstract}

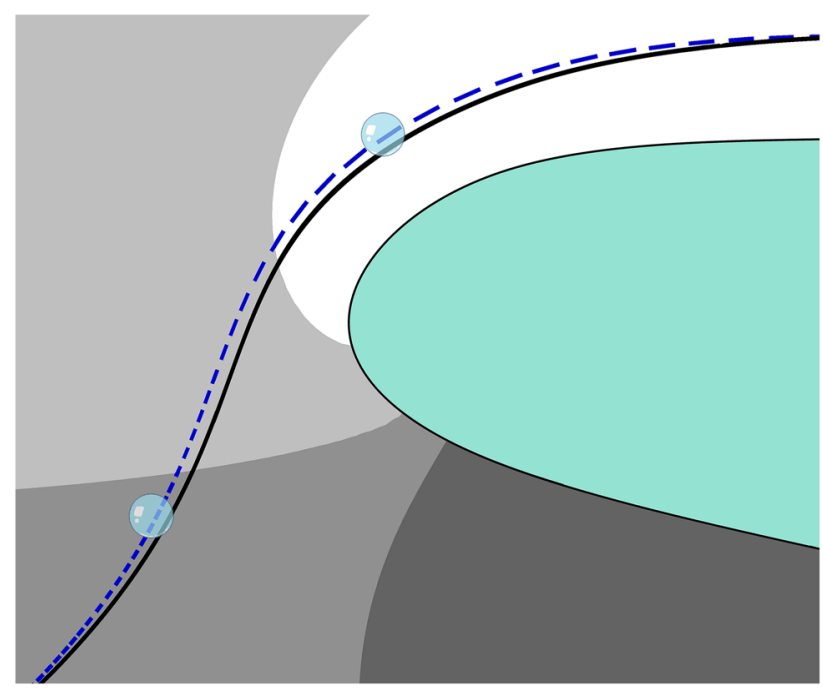

Marthijn Tuinstra

marthijn.tuinstra@nlr.nl

$1 \quad$ Vertical Flight and Aeroacoustics, Royal Netherlands Aerospace Centre (NLR), Amsterdam, The Netherlands

2 Faculty of Aerospace Engineering, Delft University of Technology, Delft, The Netherlands

\section{Introduction}

Particle image velocimetry (PIV) is generally deemed as a non-intrusive technique for measuring the instantaneous flow velocity, in contrast to probe-based techniques. However, the tracer particles must follow the flow faithfully for accurate measurements, a crucial requirement often taken for granted. 
Analysis of particle tracking accuracy originates from the pioneering work of Stokes (1851), in which he derived the equation for the drag force of a rigid sphere in a viscous flow at very small Reynolds number values, the so-called Stokes law. A few decades later, Boussinesq (1885a, b) and Basset (1888a, b) expanded the Stokes' analysis to the more general case of unsteady motion, with the particle velocity being an arbitrary function of time, however, still under creeping flow conditions. There have been several works following Stokes, Boussinesq and Basset, attempting to relax the assumption of creeping flow to achieve a more general dynamic equation that would describe the motion of particles with small, yet finite, particle Reynolds number. Maxey and Riley (1983) give a review of the most relevant developments in the century following Boussinesq's and Basset's papers and provide a more generic equation for a small rigid sphere, including the forces due to non-uniform flow. However, most useful equations up to date still rely on semi-empirical corrections (especially for the drag coefficient), resultant from experiments, simulations and analytical expansions of asymptotic solutions (Mei 1996; Magnaudet 1997; Michaelides 1997; Loth and Dorgan 2009).

Based on analytical models, Melling (1997) analysed the tracking characteristics of typical particles used for PIV, restricted to the case of particles much heavier than air, in which the Boussinesq-Basset's equation is greatly simplified. From these theoretical considerations, it was concluded that typical PIV particles with density in the range of $1000-4000 \mathrm{~kg} / \mathrm{m}^{3}$ should not exceed $1 \mu \mathrm{m}$ in diameter for a frequency response of $10 \mathrm{kHz}$. Particle relaxation length and time were obtained across shock waves by Scarano and van Oudheusden (2003) in the supersonic regime and by Schrijer et al. (2006) up to Mach 7, returning a response time of submicron $\mathrm{TiO} 2$ particles in the range of $2 \mu \mathrm{s}$. A systematic study of micron-sized flow tracers conducted by Ragni et al. (2010), comprising several particles used for PIV, reported a time response of $2 \mu$ s for di-ethyl-hexylsebacat (DEHS) droplets of $1 \mu \mathrm{m}$ median diameter. Based on the study of Samimy and Lele (1991), it can be inferred that velocity measurements with micrometre particles of $2 \mu$ s time response result in errors smaller than $2 \%$ for flow frequencies up to $100 \mathrm{kHz}$.

A known drawback of micron-sized tracers is the limited amount of light scattered from laser illumination, due to the small scattering cross-section (Adrian and Yao 1985), rendering them optically ineffective when the scale of the experiment is increased. For instance, three-dimensional measurements with tomographic PIV seldom exceed $100 \mathrm{~cm}^{3}$ (Scarano 2013).

The amount of light scattering can be increased without significantly compromising the tracing fidelity by adopting larger particles with reduced weight, such that the particles become approximately neutrally buoyant. If the velocity gradients at the particle scale are negligible, spherical neutrally buoyant particles follow the flow perfectly (Mei 1996). In air, a particle of neutral density is obtained through the generation of soap bubbles filled with a light gas, usually helium (Hale et al. 1969; Bosbach et al. 2009) of about $0.5 \mathrm{~mm}$ diameter. Although particles cannot follow velocity fluctuations occurring at turbulent length scales smaller than their diameter, even if neutrally buoyant (Xu and Bodenschatz 2008), submillimetre helium-filled soap bubbles (HFSB) have been shown to accurately retrieve the mean velocity and turbulent fluctuations for wall distances larger than twice their diameter (Faleiros et al. 2018). The use of submillimetre HFSB has been reported to reflect $10^{4}$ to $10^{5}$ times more light than micrometre particles (Caridi 2018). In the past two decades, HFSB have been applied to several PIV experiments at metre scale: aircraft cabins (Bosbach et al. 2009), wind turbines (Caridi et al. 2016), thermal plumes (Huhn et al. 2017), aeronautics (Sciacchitano et al. 2018), full-scale cyclists (Jux et al. 2018; Terra et al. 2019; Spoelstra et al. 2019), aeroacoustics (Lima Pereira et al. 2020) and bird flight (Usherwood et al. 2020).

HFSB can be produced as neutrally buoyant tracers by careful control of the supply rate of their constituents. However, small deviations from neutral buoyancy always occur in practice, leading to a slip velocity between the particle and the flow. Kerho and Bragg (1994) were the first to attempt to characterize the tracing fidelity of HFSB for aerodynamic experiments, measuring the slip velocity along the bubble trajectories in the flow around an airfoil. Errors of up to $10 \%$ of $U_{\infty}$ were ascribed to a bubble generation process biased towards lighter-than-air particles. Based on dimensional considerations, Scarano et al. (2015) devised a practical approach for measuring the HFSB time response in the stagnation region of a cylinder. Although not explicitly mentioned, comparison to the equation of particle motion, as given by Mei (1996), shows that the approach assumes approximately equal acceleration between particle and fluid. Applying the procedure experimentally, a mean time response of approximately $10 \mu$ s was measured for submillimetre HFSB. The methodology was followed by many authors for the study of HFSB tracing fidelity (Morias et al. 2016; Gibeau and Ghaemi 2018; Faleiros et al. 2019; Gibeau et al. 2020). Morias et al. (2016) reported measurements of the standard deviation of HFSB time response of $40-50 \mu$ s, exceeding its mean. They also observed that the bubbles could be produced in two regimes, bubbling and jetting, with only the former resulting in bubbles of uniform size. Faleiros et al. (2019) performed a rigorous assessment of such regimes for several input values of helium, air and soap volume flow rates, resulting in general guidelines for the generation of HFSB in the bubbling regime, and the control of bubble size, density and production rate. A mean time response below $20 \mu$ s was found, corresponding to density 
deviations smaller than $10 \%$. The time response dispersion of about $40 \mu$ s was found to be independent of the regime of production. Gibeau and Ghaemi (2018) and Gibeau et al. (2020) performed similar experiments and also obtained mean time responses below $10 \mu$ s for bubbles approaching neutral buoyancy; however, standard deviations as high as $180 \mu$ s were reported. The dispersion of the physical properties of HFSB tracers could therefore represent a more critical phenomenon than matching the ensemble average density of the tracers to the air density. Furthermore, the tracing fidelity of HFSB has also been verified in isotropic turbulence (Qureshi et al. 2008; Bourgoin et al. 2011) and wall-bounded turbulent flows (Faleiros et al. 2018), with the results showing accurate measurements of turbulent stresses, even for air-filled soap bubbles.

In most PIV experiments, it is desirable to know the expected particle slip velocity for estimating the measurement uncertainty. Although the method devised by Scarano et al. (2015) is adequate for time response estimation when the particle acceleration does not depart significantly from that of the surrounding fluid, it may not hold for many practical situations. For instance, in the work of Morias et al. (2016), when a 30\% heavier-than-air HFSB approaches the cylinder stagnation point (within $10 \%$ of the cylinder diameter from the leading edge), the mean particle deceleration is up to twice as large as that of the air flow. The HFSB slip velocity has not been investigated when the particle acceleration deviates considerably from that of the flow. In fact, current research on HFSB focused mostly on the generation of neutrally buoyant bubbles rather than the estimation of the slip velocity of nearly neutrally buoyant tracers. The timeline in Fig. 1 summarizes the main investigations on HFSB tracing fidelity, focused on its quantification and control.

The present work surveys the available models for estimating the slip velocity of nearly neutrally buoyant particles and provides a generalized model within a particle flow parameter space that is typical of large-scale PIV experiments conducted with HFSB. The approach expands from simple predictions of HFSB flow tracing accuracy based on the Stokes regime, or other similar simplifications that neglect the unsteady forces, towards a model that incorporates the latter and allow for large acceleration differences.

For this purpose, the role of nonlinear terms in the equation of motion is examined. The slip velocity of HFSB cannot be simply estimated from a single time response parameter, as in the case of heavy small particles (Melling, 1997), because the unsteady forces also become important and bring additional complexity to the analysis. Although the slip velocity can be promptly calculated by realizing a numerical simulation (similar to that carried out in this paper), the intention is to provide means for judging the experimental velocity errors without the need of such timeconsuming computations.

This is performed by analysing the motion of nearly neutrally buoyant particles through numerical simulations in a rectilinear oscillatory flow and in the flow around an airfoil with focus on the high-acceleration region in the vicinity of the leading edge. From these analyses, an extension of the current methods used to estimate the slip velocity is proposed.

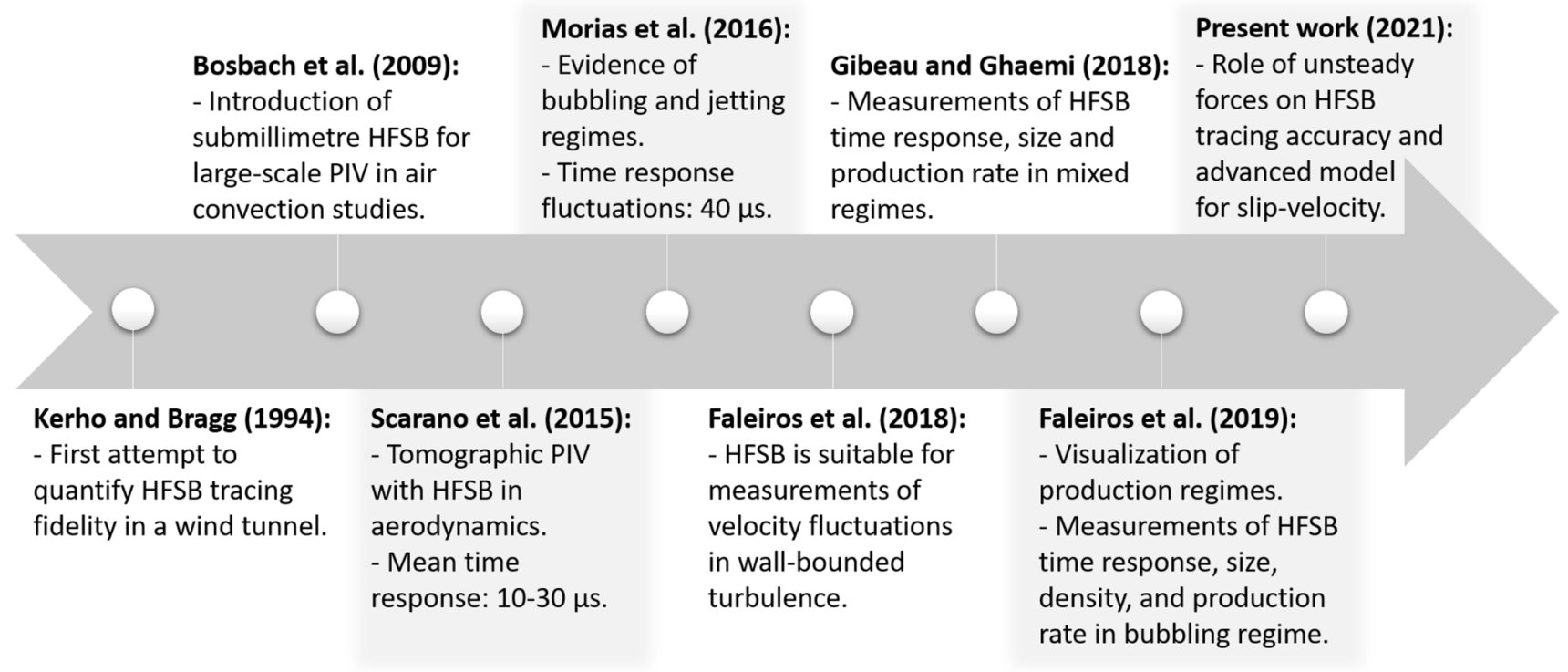

Fig. 1 Timeline of main contributions regarding control of HFSB generation (focused on the generation of neutrally buoyant bubbles) and quantification of their tracing fidelity. A complete overview of
HFSB generation would also have to include the works of Hale et al. (1969), Okuno et al. (1993), Caridi et al. (2016), Gibeau et al. (2020), among others 
Additionally, a set of experiments in a large-scale aerodynamic wind tunnel is conducted to examine the tracer behaviour of HFSB at high-Reynolds-number flows, under similar conditions than the simulations. The potential of the proposed model to assess PIV measurement errors using HFSB is demonstrated via application to experimental data, from which the HFSB density is retrieved, helping to identify the source of velocity errors. In addition, the effects of tracing fidelity of PIV seeding (Melling 1997; Scarano and van Oudheusden 2003; Schrijer et al. 2006; Lazar et al. 2010; Ragni et al. 2010).

In the case of large nearly neutrally buoyant particles, the unsteady forces become relevant and the Boussinesq-Basset's equation with the corrections for finite $\mathrm{Re}_{p}$ must be applied. The full unsteady equation of motion as proposed by Mei (1996) is:

$$
\begin{aligned}
& \text { Added - mass } \\
& \hat{\mathrm{d}} \frac{\overrightarrow{\hat{u}}_{p}}{\mathrm{~d} \hat{t}}=\overbrace{(\hat{\rho}-1) \vec{g}}^{\text {Buoyancy force }}+\overbrace{\frac{\mathrm{D} \overrightarrow{\hat{u}}}{\mathrm{D} \hat{t}}}^{\text {Undist. fluid stresses }}+\overbrace{\frac{1}{2}\left(\frac{\mathrm{D} \hat{\hat{u}}}{\mathrm{D} \hat{t}}-\frac{\mathrm{d} \overrightarrow{\hat{u}}_{p}}{\mathrm{~d} \hat{t}}\right)}^{\text {force }}-\overbrace{\frac{\hat{\rho}}{\tau_{p} f\left(\operatorname{Re}_{p}\right) \vec{u}_{\text {slip }}}-\frac{\hat{\rho}}{\tau_{p}} \int_{t^{-}}^{t} K(t-\tau) \frac{\mathrm{d} \overrightarrow{\hat{u}}_{\text {slip }}}{\mathrm{d} \hat{\tau}} \mathrm{d} \hat{\tau}}^{\text {Quasi - steady Drag }},
\end{aligned}
$$

the HFSB density dispersion, herein proposed as the main source of the previously measured time response dispersions (Morias et al. 2016; Faleiros et al. 2019), are also investigated.

\section{Background}

\subsection{The equation of particle motion}

For a spherical heavy particle, the equation of motion, neglecting buoyancy force, reads as (Melling 1997):

$\frac{\mathrm{d} \vec{u}_{p}}{\mathrm{~d} t}=-\frac{\vec{u}_{\text {slip }}}{\tau_{p}}$,

where $\vec{u}_{\text {slip }}=\vec{u}_{p}-\vec{u}$ is the slip velocity, $\vec{u}_{p}$ and $\vec{u}$ and are the particle and fluid velocity, respectively, $\tau_{p}=\rho_{p} \mathrm{~d}_{p}^{2} / 18 \mu$ is the particle time response, $\rho_{p}$ is the particle density, $d_{p}$ is the particle diameter and $\mu$ is the fluid dynamic viscosity. The term on the right-hand side is commonly referred to as Stokes' drag (Stokes 1851). Equation (1) is exact for $\operatorname{Re}_{p} \ll 1$ and $\rho_{p} \gg \rho$, where $\operatorname{Re}_{p}=\mathrm{d}_{p}\left|\vec{u}_{\text {slip }}\right| / \nu$ is the particle Reynolds number, $\rho$ is the fluid density and $v$ is the fluid kinematic viscosity. Although the applicability of Eq. (1) seems limited, it describes accurately the motion of micrometre heavy particles in air, and it is commonly used for evaluating the where $\hat{\rho}=\rho_{p} / \rho$ is the particle-to-fluid density ratio ( $\hat{\text {. }}$ is used throughout the paper for representing normalized variables), $\vec{g}$ is the gravitational acceleration, $\phi\left(\operatorname{Re}_{p}\right)$ is an empirical relation to correct for deviations from the Stokes' drag law due to a finite $\operatorname{Re}_{p}, K(t-\tau)$ is the history force kernel, $\mathrm{d} / \mathrm{d} t=\partial / \partial t+\vec{u}_{p} \cdot \nabla$ is the time derivative on the particle trajectory and $\mathrm{D} / \mathrm{D} t=\partial / \partial t+\vec{u} \cdot \nabla$ is the time derivative evaluated on the trajectory of fluid elements around the particle. Rigorously, the lower limit of integration of the history force is $-\infty$. For simulation purposes, $t^{-}$is the instant right before the particle is introduced into the flow. Furthermore, it is noted that the buoyancy force is usually negligible in external aerodynamic experiments, given that the acceleration due to the flow kinematics substantially exceeds the gravitational term, and is henceforth omitted.

In the study of aerodynamic flows, normalization of the equation of particle motion is usually based on a chosen characteristic velocity $U_{0}$ and length $L_{0}$, with the reference timescale being derived from the latter. For instance, in airfoil flows the free stream velocity and the airfoil chord (or airfoil thickness) are typical choices. Such normalization is convenient in Eq. (1), as it results in a single non-dimensional parameter, the so-called Stokes number $S_{k} \equiv \tau_{p} U_{0} / L_{0}$. Normalization of Eq. (2) based on $U_{0}$ and $L_{0}$ leads, however, to three non-dimensional parameters, $S_{k}, \hat{\rho}$ and $\mathrm{Re}_{p}$, increasing the complexity of the particle tracing fidelity analysis. Alternatively, Eq. (2) may be normalized based on a chosen reference velocity $U_{0}$ and the viscous timescale $t_{0} \equiv \mathrm{d}_{p}^{2} / v$ :

$$
\begin{aligned}
& \text { Undist. } \\
& \text { fluid Added - mass } \\
& \text { stresses force } \\
& \hat{\rho} \frac{\mathrm{d} \overrightarrow{\hat{u}}_{p}}{\mathrm{~d} \hat{t}}=\overbrace{\frac{\mathrm{D} \overrightarrow{\hat{u}}}{\mathrm{D} \hat{t}}}+\frac{1}{2} \overbrace{\left(\frac{\mathrm{D} \overrightarrow{\hat{u}}}{\mathrm{D} \hat{t}}-\frac{\mathrm{d} \overrightarrow{\hat{u}}_{p}}{\mathrm{~d} \hat{t}}\right)}^{\text {Quasi - steady Drag }}-\overbrace{18 \phi\left(\operatorname{Re}_{p}\right) \overrightarrow{\hat{u}}_{\text {slip }}}-18 \overbrace{\int_{\hat{t}^{-1}}^{\hat{t}} K(\hat{t}-\hat{\tau}) \frac{\mathrm{d} \overrightarrow{\hat{u}}_{\text {slip }}}{\mathrm{d} \hat{\tau}} \mathrm{d} \hat{\tau}},
\end{aligned}
$$


where the history force kernel may be calculated using the approximation (Mei 1994)

$K(\hat{t}-\hat{\tau}) \approx\left\{[4 \pi(\hat{t}-\hat{\tau})]^{\frac{1}{4}}+\left[\frac{\pi(\hat{t}-\hat{\tau})^{2} \operatorname{Re}_{p}^{3}}{\left(0.75+0.105 \operatorname{Re}_{p}\right)^{3}}\right]^{\frac{1}{2}}\right\}^{-2}$,

with $\operatorname{Re}_{p}$ evaluated at $\hat{t}-\hat{\tau}$. Notice that $\hat{\rho}$ and $\operatorname{Re}_{p}$ are the only non-dimensional parameters in Eqs. (3) and (4). Furthermore, although $\operatorname{Re}_{p}$ represents the actual particle Reynolds number, often it is desirable to specify a reference Reynolds number that is independent of the slip velocity. For this purpose, a diameter-based Reynolds number $\operatorname{Re}_{d} \equiv U_{0} \mathrm{~d}_{p} / v$ is defined, such that $\operatorname{Re}_{p}=\operatorname{Re}_{d}\left|\overrightarrow{\hat{u}}_{\text {slip }}\right|$.

\subsubsection{Drag correction}

Several empirical relations have been proposed for the drag correction term $\phi\left(\operatorname{Re}_{p}\right)=C_{d} / C_{d 0}($ Clift et al. 1978), where $C_{d}$ is the particle drag coefficient and $C_{d 0}=24 / \operatorname{Re}_{p}$ is the drag coefficient of a rigid sphere given by the Stokes' law $\left(\operatorname{Re}_{p} \ll 1\right)$. A commonly used expression given by Schiller and Naumann (1933) for which the drag coefficient is accurate within $5 \%$ for $\operatorname{Re}_{p} \leq 800$ :

$\phi\left(\operatorname{Re}_{p}\right)=1+0.15 \operatorname{Re}_{p}^{0.687}$.

More accurate approximations were compiled by Clift et al. 1978, for different $\operatorname{Re}_{p}$ ranges:

$\begin{array}{ll}\phi\left(\operatorname{Re}_{p}\right)=1+\frac{3}{16} \operatorname{Re}_{p} & \operatorname{Re}_{p} \leq 0.01 \\ =1+0.1315 \operatorname{Re}_{p}^{0.82-0.05 w} & 0.01<\operatorname{Re}_{p} \leq 20 \\ =1+0.1935 \operatorname{Re}_{p}^{0.6305} & 20<\operatorname{Re}_{p} \leq 260 \\ =1.8335 \operatorname{Re}_{p}^{0.1558 w-0.1242} & 260<\operatorname{Re}_{p} \leq 1500 \\ =1.45 \times 10^{-4} \operatorname{Re}_{p}^{3.5558-0.9295 w+0.1049 w^{2}} & 1500<\operatorname{Re}_{p} \leq 12,000\end{array}$,

where $w=\log \left(\operatorname{Re}_{p}\right)$. There are different expressions for the drag coefficient in the case of a clean bubble (the flow slips along the surface) or due to particle deformation (Clift et al. 1978; Magnaudet and Eames 2000; Loth 2008). In this study, it is assumed that the particles are rigid non-deformable spheres, in which the no-slip condition at the surface applies. A soap bubble of $0.5 \mathrm{~mm}$ diameter in air maintains its sphericity (less than $10 \%$ difference in size between the two spheroid semi-axes) for slip velocities up to about $10 \mathrm{~m} / \mathrm{s}$ (Faleiros 2021). The no-slip condition applies if the surface is sufficiently contaminated with surfactants. A soap bubble can be considered fully contaminated if the contaminants concentration is larger than $10^{-2} \mathrm{~g} / 1$ (Loth 2008). The amount of surfactants used
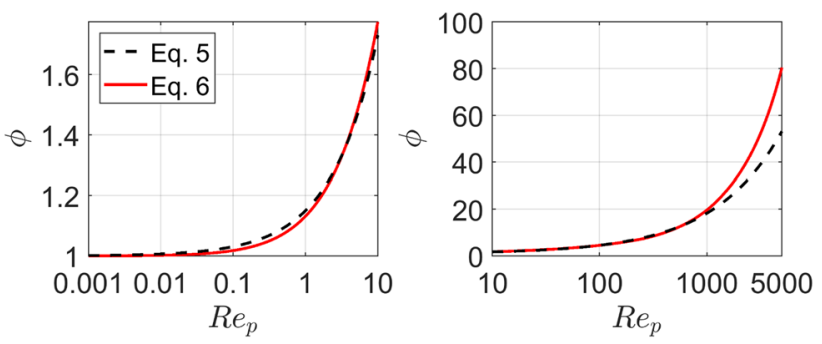

Fig. 2 Comparison of drag correction given by Eqs. (5) and (6)

to produce soap solutions for HFSB is far greater than this threshold (Hale et al. 1971).

Comparison of Eq. (5) and (6) (Fig. 2) shows that, up to $\operatorname{Re}_{p}=800$, the corrections differ less than $5 \%$ from each other. However, as $\operatorname{Re}_{p}$ surpasses this limit, Eq. (5) underestimates drag significantly, with the estimate from Eq. (6) being 50\% larger than that given by Eq. (5) at $\operatorname{Re}_{p}=5000$. In most PIV applications, Eq. (5) is sufficient, since $\operatorname{Re}_{p}=800$ represents a slip velocity of $24 \mathrm{~m} / \mathrm{s}$ for a half-millimetre particle in air. Furthermore, Fig. 2 shows that the assumption of $\phi\left(\operatorname{Re}_{p}\right)=1$ is limited to low-speed flows and small particles. The correction term is already twice this value $\left(\phi\left(\operatorname{Re}_{p}\right) \approx 2\right.$ ) for a slip velocity of $0.4 \mathrm{~m} / \mathrm{s}$ under the same conditions.

\subsubsection{Faxén terms and lift force}

Strictly speaking, the flow velocity and acceleration should be calculated as averages along the particle surface $\vec{u}_{S}$ and over its volume $\mathrm{D} \vec{u} / \mathrm{D} t_{V}$, respectively. Faxén (1922), as cited in Michaelides (1997), derived approximations to these averages using a Taylor series expansion in the particle limit $\mathrm{d}_{p} \rightarrow 0$ for $\vec{u} \approx \vec{u}_{p}$, resulting in extra terms that contain the Laplacian of the velocity (Mei 1996), the socalled Faxén terms. However, in the numerical simulations performed in this study, the flow field is derived from a potential flow solution. In an incompressible $(\nabla \cdot \vec{u}=0)$ and irrotational $(\nabla \times \vec{u}=0)$ flow, the Laplacian of the velocity is equal to zero. In fact, even in rotational flows the Faxén terms are usually negligible in comparison with the remaining terms (Mei 1996), only becoming relevant when the slip velocity approaches zero, and the effect of drag and history force have the least impact on the net force (Calzavarini et al. 2009). Therefore, they have been omitted from Eq. (2).

Additionally it is noted that the lift force has also been neglected. Shear lift or spin lift induced by shear is not relevant for the flow around an airfoil $(\vec{\omega}=\overrightarrow{0})$, only in 
the boundary layer. The influence of wall lift (Zeng et al. 2009), which exists even in a shear free flow, is only important at distances from the wall $L_{W}$ of $O\left(\mathrm{~d}_{p}\right)$ and for low $\mathrm{Re}_{p}$. From the empirical relations given by Zeng et al. (2009), as reviewed by Shi and Rzehak (2020), it is estimated that for $L_{W} / \mathrm{d}_{p}>2$ the ratio of lift to drag coefficient $C_{l} / C_{d}$ is less than $1 \%$ for $\operatorname{Re}_{p}>25$. In the simulations performed in Sect. 3.3, the particles approach the airfoil at $50<\operatorname{Re}_{p}<200$. Thus, wall lift can also be safely neglected here.

\subsection{Simplifications to the equation of particle motion}

In this section, Eq. (2) is studied under different assumptions in order to reproduce previous models available in the literature for the analysis of particle slip velocity (Sect. 2.3). Notice, however, that in numerical simulations presented in chapter 3, Eq. (2) is used as presented.

If, for the sake of the discussion, the buoyancy and history forces are neglected, Eq. (2) becomes:

$\hat{\rho} \frac{\mathrm{d} \vec{u}_{p}}{\mathrm{~d} t}=\frac{\mathrm{D} \vec{u}}{\mathrm{D} t}+\frac{1}{2}\left(\frac{\mathrm{D} \vec{u}}{\mathrm{D} t}-\frac{\mathrm{d} \vec{u}_{p}}{\mathrm{~d} t}\right)-\frac{\hat{\rho}}{\tau_{p}} \phi\left(\operatorname{Re}_{p}\right) \vec{u}_{\text {slip }}$.

In the study of micrometre heavy particles (Melling 1997) typically used for PIV, $\hat{\rho} \gg 1$ and Eq. (7) is greatly simplified to:

$\frac{\mathrm{d} \vec{u}_{p}}{\mathrm{~d} t}=-\frac{\phi\left(\operatorname{Re}_{p}\right)}{\tau_{p}} \vec{u}_{\text {slip }}$.

As $\operatorname{Re}_{p} \rightarrow 0, \phi\left(\operatorname{Re}_{p}\right) \rightarrow 1$, and Eq. (8) returns to Eq. (1). This simplified form enables analysis of the slip velocity based only on particle acceleration and time response. Other simplifications to the equation of motion have attempted to enable similar analysis without the restriction of $\hat{\rho} \gg 1$. If the added mass force is also neglected, Eq. (7) becomes:

$\hat{\rho} \frac{\mathrm{d} \vec{u}_{p}}{\mathrm{~d} t}=\frac{\mathrm{D} \vec{u}}{\mathrm{D} t}-\frac{\hat{\rho}}{\tau_{p}} \phi\left(\operatorname{Re}_{p}\right) \vec{u}_{\text {slip }}$.

In addition, for flow regions where the particle has approximately the same acceleration as the flow, the slip velocity may be written in a similar fashion to Eq. (1):

$\vec{u}_{\text {slip }}=-\tau_{p}^{*} \frac{\mathrm{D} \vec{u}}{\mathrm{D} t}=-\tau_{p}^{*} \frac{\mathrm{d} \vec{u}_{p}}{\mathrm{~d} t}$,

where

$\tau_{p}^{*} \equiv \frac{\left(\rho_{p}-\rho\right) \mathrm{d}_{p}^{2}}{18 \mu \phi\left(\operatorname{Re}_{p}\right)}$ is the particle time response typically used in the study of HFSB tracing fidelity (Scarano et al. 2015; Morias et al. 2016; Faleiros et al. 2019; Gibeau et al. 2020).

Similarly to Eq. (1), Eq. (10) provides an operational approach to the measurement of the particle time response through the simultaneous measurement of the slip velocity and the flow acceleration. Furthermore, if the particle size is also measured, e.g. by estimation of the bubble glare points distance (Morias et al. 2016; Faleiros et al. 2019), Eq. (11) yields an estimate of the particle density, a notably difficult parameter to measure.

In comparison with $\tau_{p}, \tau_{p}^{*}$ is proportional to the density difference $\rho_{p}-\rho$, representing more appropriately the time response of nearly neutrally buoyant particles. However, it is emphasized that basing the analysis of HFSB tracing fidelity only on $\tau_{p}^{*}$ is an oversimplification that is only valid if the particle acceleration is approximately equal to the fluid acceleration.

\subsection{The slip velocity in an oscillating flow field}

It is instructive to first consider the case of a spherical particle immersed in a rectilinear oscillating flow field. The respective velocities are given by $u_{p}(t)=\tilde{u}_{p}(\omega) e^{-i \omega t}$ and $u(t)=\tilde{u}(\omega) e^{-i \omega t}$, where $\tilde{u}_{p}(\omega)$ and $\tilde{u}(\omega)$ are the particle and flow amplitudes of the oscillation, and $\omega=2 \pi f$ is the angular frequency.

Assuming negligible history force and added mass and $\phi\left(\operatorname{Re}_{p}\right)=1$, which is acceptable for low $\operatorname{Re}_{p}$, substitution of the particle and flow oscillating velocity and acceleration in Eq. (9) gives:

$-i \omega \tilde{u}_{p} \hat{\rho}+\hat{\rho} \frac{\tilde{u}_{p}}{\tau_{p}}=-i \omega \tilde{u}+\hat{\rho} \frac{\tilde{u}}{\tau_{p}}$.

From Eq. (12), the particle tracing fidelity can be analysed from the ratio of the slip velocity to the flow velocity amplitudes:

$u_{R} \equiv \frac{\left(\tilde{u}_{p}-\tilde{u}\right)}{\tilde{u}}, u_{R}\left(\omega, \tau_{p}, \hat{\rho}\right)=\frac{i(\hat{\rho}-1) \omega \frac{\tau_{p}}{\hat{\rho}}}{1-i \omega \tau_{p}}$.

Following Mei (1996), $u_{R}\left(\omega, \tau_{p}, \hat{\rho}\right)$ may be rewritten as a function of $\hat{\rho}$ and $\epsilon$ only,

$u_{R}(\epsilon, \hat{\rho})=\frac{i \frac{4}{9}(\hat{\rho}-1) \epsilon^{2}}{1-i \frac{4}{9} \hat{\rho} \epsilon^{2}}$,

where $\epsilon$ is a non-dimensional parameter with similar significance to the Stokes number: 
$\epsilon \equiv \sqrt{\frac{\omega \mathrm{d}_{p}^{2}}{8 v}}$.

It can also be easily verified that if the added mass had been included, Eq. (7), then the amplitude ratio becomes:

$u_{R}(\epsilon, \hat{\rho})=\frac{i \frac{4}{9}(\hat{\rho}-1) \epsilon^{2}}{1-i \frac{4}{9}\left(\hat{\rho}+\frac{1}{2}\right) \epsilon^{2}}$.

Furthermore, the analysis can be expanded by considering an approximation to the history force in the oscillating flow field for $\epsilon \gg 1$ (Mei 1996):

$\frac{\hat{\rho}}{\tau_{p}} \int_{t^{-1}}^{t} K(t-\tau) \frac{\mathrm{d}\left(\vec{u}-\vec{u}_{p}\right)}{\mathrm{d} \tau} \mathrm{d} \tau \approx \frac{\hat{\rho}}{\tau_{p}} \epsilon(1-i)\left(\tilde{u}-\tilde{u}_{p}\right) e^{-i \omega t}$.

Including added mass and history force, the amplitude ratio becomes:

$u_{R}(\epsilon, \hat{\rho})=\frac{i \frac{4}{9}(\hat{\rho}-1) \epsilon^{2}}{1-i \frac{4}{9}\left(\hat{\rho}+\frac{1}{2}\right) \epsilon^{2}+\epsilon(1-i)}$,

where the history force is represented by the term $\epsilon(1-i)$.

For the purpose of illustration (Fig. 3), the tracing behaviour of lighter- $(\hat{\rho}=0.5)$ and heavier-than-air $(\hat{\rho}=1.5)$ particles is modelled on the basis of Eq. (14), which neglects both added mass and history forces, and Eq. (18), in which both forces are included. The particle motion modelled by Eq. (14) (Fig. 3, left) exhibits a positive phase lag and some degree of amplitude modulation for the heavier-thanair conditions. In the lighter-than-air case, the situation is reversed: the amplitude of the velocity oscillations exceeds that of the flow and a negative phase lag is observed. These observations are in accordance with numerical simulations performed by Müller et al. (2001). The situation remains qualitatively unaltered when the particle motion is modelled

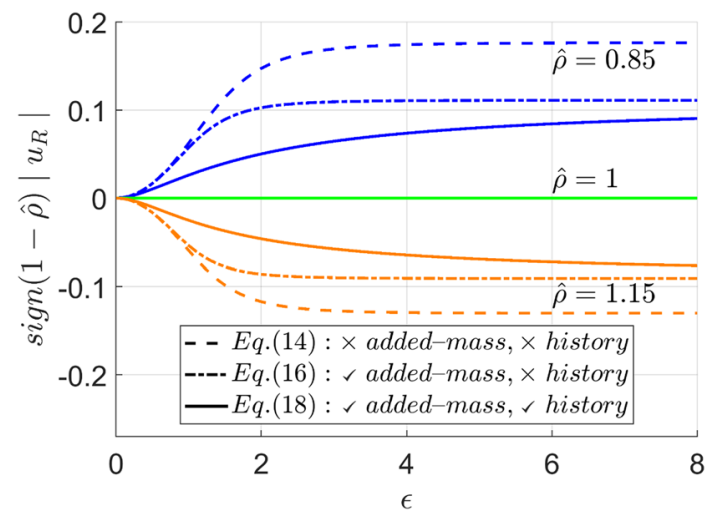

Fig. 4 Slip velocity amplitude modulation modelled by Eqs. (14), (16) and (18). The absolute value $\left|u_{R}\right|$ is multiplied by $\operatorname{sign}(1-\hat{\rho})$ for clarity of visualization. Lighter-than-air particles: positive side of the vertical axis. Heavier-than-air particles: negative side

by Eq. (18), including the added mass and history force term (Fig. 3, right). However, a notable contraction of the discrepancy between the particle and the fluid motion is observed. This is because the added mass and history forces are proportional to the slip acceleration and act as restoring forces, reducing the acceleration difference, and resulting in a higher tracing fidelity. Analysis of the spectral behaviour of the slip velocity amplitudes (Fig. 4) gives an overview of the role of the unsteady forces. In general, including the added mass force as well as of the history force results in reduced slip velocities for all $\hat{\rho}$ and $\epsilon>1$.

Furthermore, note that the slip velocity amplitude converges to a finite value for large $\epsilon$. At large oscillation frequencies, the acceleration-driven terms are dominant, as $|\mathrm{d} u / \mathrm{d} t| /|u|=\omega$. Therefore, the Stokes' drag force, being slip velocity-driven, becomes negligible at high frequencies in comparison with the other forces. When added mass and history force are neglected, it is found from Eq. (14) that $\left|u_{R}\right| \rightarrow(1-\hat{\rho}) / \hat{\rho}$ for $\epsilon \rightarrow \infty$. If the added mass term is included, then $\left|u_{R}\right| \rightarrow(1-\hat{\rho}) /\left(\hat{\rho}+\frac{1}{2}\right)$ for $\epsilon \rightarrow \infty$. This is
Fig. 3 Particle velocity in an oscillating flow field. Simulated conditions are representative of aerodynamic experiments using HFSB $\left(f=100 \mathrm{~Hz}, v=15 \mathrm{~mm}^{2} / \mathrm{s}\right.$ and $\mathrm{d}_{p}=0.5 \mathrm{~mm}$ ), with the exception of the density ratio that is accentuated to emphasize the particle behaviour. Left: Eq. (14), neglecting added mass and history force. Right: Eq. (18), including both forces. Fluid velocity: solid blue line. Fluid acceleration: solid red line
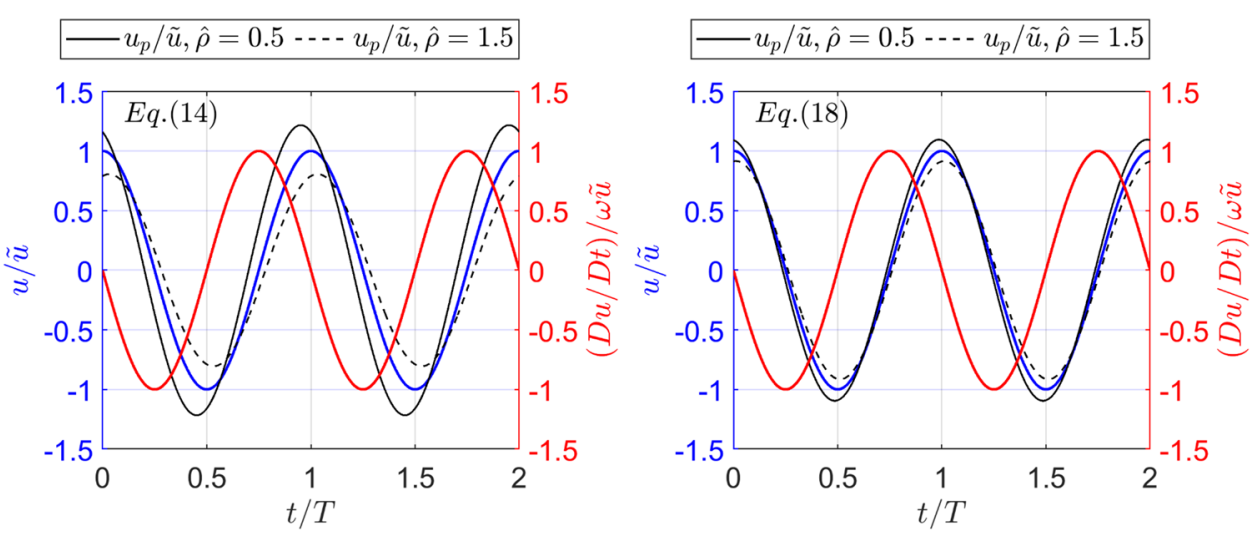
true for both Eq. (16) and (18), because the history force scales with $\epsilon$-while the other forces scale with $\epsilon^{2}$-not contributing, therefore, to $\left|u_{R}\right|$ in the limit of $\epsilon \rightarrow \infty$. The latter cannot be observed in Fig. 4 due to the short range of $\epsilon$ that is plotted.

This analysis shows the importance of including the unsteady forces in the study of nearly neutrally buoyant particle motion. Estimates of tracing fidelity based only on Stokes' drag become increasingly conservative as the frequency of the flow fluctuation increases, yielding overestimated predictions of the slip velocity. In addition, the estimates from Eq. (18) should also be considered conservative due to the assumption of $\phi\left(\operatorname{Re}_{p}\right)=1$. The slip velocity is further reduced when drag correction is considered-see Eq. (8) and (10).

\section{The slip velocity: from sinusoids to the flow around an airfoil}

In the case of a particle moving around an object, the analysis of the slip velocity becomes geometry dependent and, therefore, case-specific. The problem can be generalized by making an analogy between the velocity modulation of a particle along its trajectory and that occurring in a sinusoid flow. Particle trajectories represent finite oscillations that can be approximated by a sinusoid of defined amplitude and frequency, within a finite time interval. This can be visualized in Fig. 5 for the flow around a cylinder. As the particle moves around the object, the streamwise component of velocity oscillates from a minimum value upstream, to a maximum on top of the cylinder, returning to the same minimum value downstream. In addition, the transverse component fluctuates in a similar fashion, but as if " $90^{\circ}$ out of phase", that is, the transverse component is maximum/minimum while the streamwise component is zero, and vice versa.

Since the two components of velocity are orthogonally out of phase, the dynamics experienced by a particle travelling around an object are analogous to that in a complex sinusoid. Thus, the streamwise and transverse components of velocity can be thought of as the "real" and "imaginary" parts of the flow. This analogy allows taking advantage of the property that the angular frequency may be obtained locally through the ratio between the absolute values of acceleration and velocity. Thus, a local frequency $f$ and phase $\beta$ can be defined as:

$f=\left|\frac{\mathrm{D} \vec{u}}{\mathrm{~d} t}\right| / 2 \pi \tilde{u}, \beta=a \tan \frac{v}{u-U_{\infty}}$

where $\tilde{u}=\sqrt{\left(u-U_{\infty}\right)^{2}+v^{2}}$ is the amplitude of this oscillation. The free stream velocity is subtracted from the velocity components, to ensure that the particle velocity oscillates around zero.

The flow frequency and phase defined in Eq. (19) are shown in Fig. 6. The approximation proposed is most adequate in the region $-\pi / 2<\beta<\pi / 2$. Within this phase band, covering half cycle of a sinusoid, the frequency along a streamline remains approximately constant, similarly to a complex sinusoid.

The hypothesis set forth is that through the simple calculation of $f$ and $\beta$ in the flow around an object, the slip velocity may be approximated through predictions obtained from a sinusoid flow at the same frequency and phase. The slip velocity components may then be obtained as

$\vec{u}_{\text {slip }}=u_{\text {slip }} \vec{e}_{x}+v_{\text {slip }} \vec{e}_{y}$,

$u_{\text {slip }}=\tilde{u}_{p} \cos \beta_{p}-\tilde{u} \cos \beta, v_{\text {slip }}=\tilde{u}_{p} \sin \beta_{p}-\tilde{u} \sin \beta$,
Fig. 5 Flow streamlines around a cylinder. The cylinder diameter $D$ is used for normalization
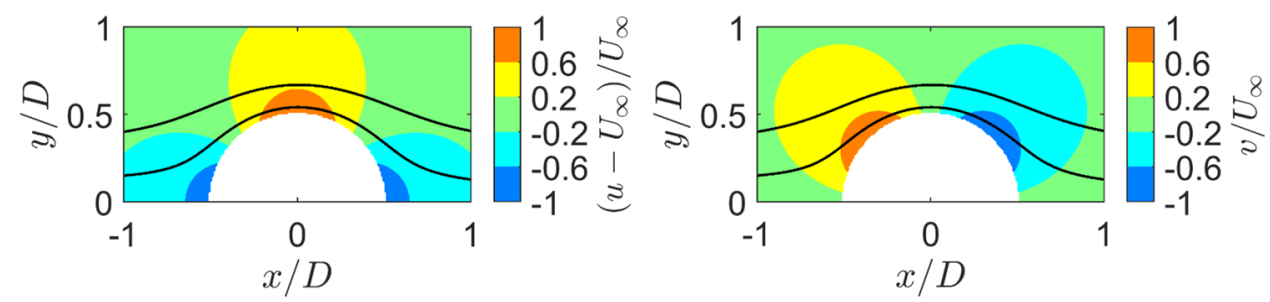

Fig. 6 Frequency (left) and phase (right) obtained from Eq. (19)
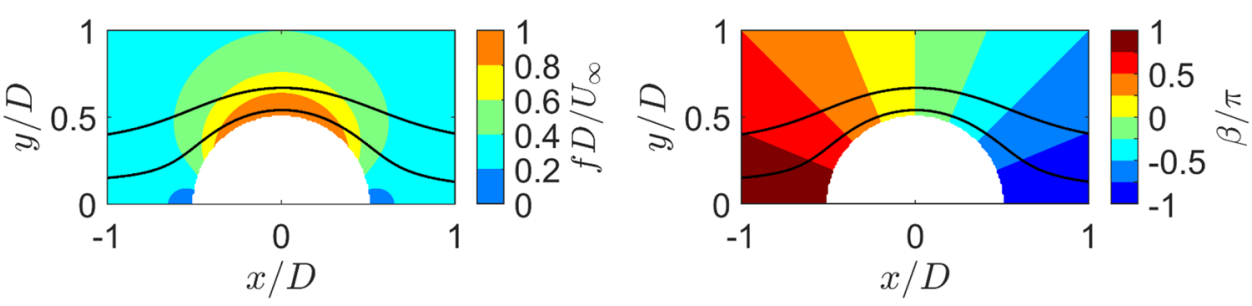
Table 1 Input parameters used in the simulations. The frequency range is wider for $\operatorname{Re}_{d} \geq 2000$

\begin{tabular}{lll}
\hline$\hat{f}$ & $\operatorname{Re}_{d}$ & $\hat{\rho}$ \\
\hline$[0.05,100]$ & {$[10,1000]$} & $\{0.7,0.8,0.9,0.95,0.99$, \\
& & $1.01,1.05,1.1,1.2$, \\
& $1.3\}$ \\
{$[0.05,2000]$} & {$[2000,50,000]$} & \\
\hline
\end{tabular}

reducing the problem to finding the amplitude modulation $\tilde{u}_{p} / \tilde{u}$ and the phase shift $\beta_{s}=\beta_{p}-\beta$ for a given particle and flow frequency.

\subsection{Numerical simulation set-up}

The drag correction term $\phi\left(\operatorname{Re}_{p}\right)$ is not considered in Sect. 2.3, and the history force was obtained through an approximation only valid for $\epsilon \gg 1$. In this section, the rectilinear oscillating flow field is simulated using the full equations of motion, i.e. Equations (3), (4) and (6), with the purpose of finding an empirical relation suitable for assessing the slip velocity of HFSB tracers, depending on $\hat{\rho}, \operatorname{Re}_{d}$ and $\hat{f}=f \mathrm{~d}_{p}^{2} / \nu$. The simulated input parameters (Table 1 ) include a wide range of applications. Considering slip velocities from 0.1 to $10 \%$ of the reference velocity (wave amplitude), $\operatorname{Re}_{p}$ varies from the Stokes regime $\left(\operatorname{Re}_{p}=0.01\right)$ to regions extending that expected for HSFB in subsonic aerodynamics $\left(\operatorname{Re}_{p}=5000\right)$. The frequency range is selected such that the asymptotic convergence of the velocity amplitude becomes apparent (see Fig. 4). Note that this occurs at higher frequencies as $\mathrm{Re}_{d}$ increases. The density range covers $\pm 30 \%$ density deviations from neutrally buoyant particles, including particles of small density differences, down to $1 \%$, allowing the empirical fits to better capture the particle behaviour near neutral buoyancy.

Neglecting gravity force, and rearranging the terms, Eq. (3) may be rewritten for a 1D flow as:

$C \frac{\mathrm{d} \hat{u}_{p}}{\mathrm{~d} \hat{t}}+\hat{u}_{p}=\hat{u}+\hat{a}$,

$\hat{a}=\phi\left(\operatorname{Re}_{d}\right)^{-1}\left(\frac{1}{12} \frac{\mathrm{D} \hat{u}}{\mathrm{D} \hat{t}}-\int_{\hat{t}^{-}}^{\hat{t}} K(\hat{t}-\hat{\tau}) \frac{\mathrm{d} \hat{u}_{\text {slip }}}{\mathrm{d} \hat{\tau}} \mathrm{d} \hat{\tau}\right), C=\frac{2 \hat{\rho}+1}{36 \phi\left(\operatorname{Re}_{d}\right)}$,

where $\hat{u}=e^{-i \hat{\omega} \hat{t}}$. The terms $C$ and $\hat{a}$ are introduced for integration purposes only. The reference velocity $U_{0}$ is the wave amplitude, and the reference time is $t_{0}=d_{p}^{2} / \nu$. Multiplying Eq. (21) by $\exp (\hat{t} / C)$ and integrating it once yields $\hat{u}_{p}^{n+1}$, while integrating it twice yields the normalized particle position at $\hat{x}_{p}^{n+1}$ :

$$
\begin{aligned}
& \hat{u}_{p}^{n+1}=\hat{u}_{p}^{n} e^{-\Delta \hat{t} / C}+\left(\hat{u}^{n}+\hat{a}^{n}\right)\left(1-e^{-\Delta \hat{t} / C}\right), \\
& \hat{x}_{p}^{n+1}=\hat{x}_{p}^{n}+\Delta \hat{t}\left(\hat{u}^{n}+\hat{a}^{n}\right)+C\left(1-e^{-\Delta \hat{t} / C}\right)\left(\hat{u}_{p}^{n}-\hat{u}^{n}-\hat{a}^{n}\right),
\end{aligned}
$$

where the initial conditions of the integration are:

$\hat{x}_{p}(\hat{t}-\Delta \hat{t})=\hat{x}_{p}^{n}, \hat{u}_{p}(\hat{t}-\Delta \hat{t})=\hat{u}_{p}^{n}$.

The simulation is performed until the particle dynamics reach steady conditions. This is achieved by requiring that the slip velocity and the phase shift vary less than $0.1 \%$ for the duration of half a cycle.

\subsection{Velocity amplitude modulation and phase shift}

Curve fits are applied to the results of the simulation, aiming to provide a simple relation for the velocity amplitude modulation $\tilde{u}_{p} / \tilde{u}$ and phase shift $\beta_{s}$, and, consequently, the slip velocity from Eq. (20), as a function of $\hat{\rho}, \operatorname{Re}_{d}$ and $\hat{f}$. The amplitude modulation is found to be well described by the following relation:

$$
\frac{\tilde{u}_{p}}{\tilde{u}}=1+\left(\frac{1}{\hat{\rho}}-1\right) \frac{\left(0.0365|\hat{\rho}-1| \operatorname{Re}_{d}^{0.25}+0.56 \sqrt{\hat{\rho}}\right) \hat{f}}{0.0163\left|\frac{1}{\hat{\rho}}-1\right| \operatorname{Re}_{d}-5 \hat{\rho}+10+\hat{f}} .
$$

Although not valid in all limits, this expression does match a few important expectations: no amplitude modulation is observed for neutrally buoyant particles $(\hat{\rho}=1)$ or in the case of zero oscillation $(\hat{f}=0)$, i.e. $\tilde{u}_{p} / \tilde{u}=1$ in both cases. Furthermore, similarly to the slip velocity estimations given in Sect. 2.3, as $\hat{f} \rightarrow \infty$, the amplitude modulation converges to a finite value:

$\lim _{\hat{f} \rightarrow \infty} \frac{\tilde{u}_{p}}{\tilde{u}}=1+\left(\hat{\rho}^{-1}-1\right)\left(0.0365|\hat{\rho}-1| \operatorname{Re}_{d}^{0.25}+0.56 \sqrt{\hat{\rho}}\right)$.

The results of the simulations for $\tilde{u}_{p} / \tilde{u}$, including the curve fits given by Eq. (24) are shown in Fig. 7. The visual agreement of the curve fits and the simulation data suggest that the proposed equation is highly accurate within the specified conditions (Table 1). A quantitative measure of goodness of fit is provided by the statistical coefficient of determination $R^{2}$ :

$R^{2} \equiv 1-\left(S S_{\text {res }} / S S_{\text {tot }}\right)$,

where the total $\left(S S_{\text {tot }}\right)$ and the residual $\left(S S_{\text {res }}\right)$ sum of squares are defined as: 
Fig. 7 Estimated amplitude modulation (solid lines) from Eq. (24). Markers: simulated data points

Fig. 8 Estimated amplitude modulation (solid lines) from Eq. (28). Markers: simulated data points
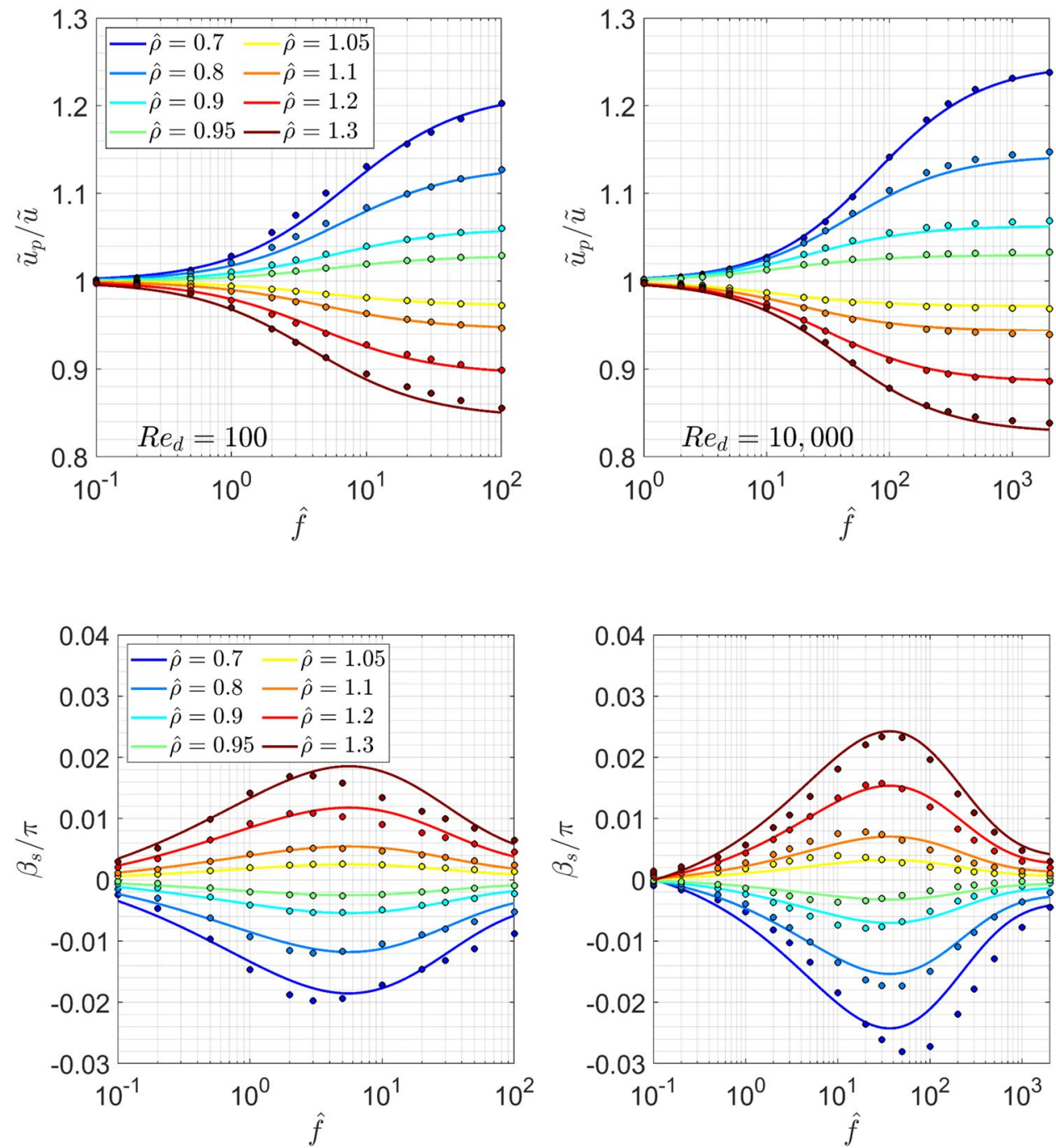

$S S_{\text {tot }} \equiv \sum_{i}\left(z_{i}-z\right)^{2}, S S_{\text {res }} \equiv \sum_{i}\left(z_{i}-F_{i}\right)^{2}$,

where $z$ is the data being fitted and $F$ is the result given by the curve fit. In general, the results demonstrate an accurate prediction of the amplitude modulation with $R^{2}>0.98$.

The phase shift is more challenging to represent with a general empirical relation, and a less accurate fit is accepted to allow generalization (Fig. 8). The best empirical relation obtained reads as:

$\beta_{\mathrm{s}}=(\hat{\rho}-1)\left[0.04-|\hat{\rho}-1|^{0.15}\left(\mathrm{e}^{\left(1.9 \mathrm{Re}_{\mathrm{d}}^{0.035}-2.9\right) \sqrt{\hat{\mathrm{f}}}}-0.9 \mathrm{e}^{-\left(1.356 \mathrm{Re}_{\mathrm{d}}^{-0.055}-0.7\right) \sqrt{\hat{\mathrm{f}}}}\right)\right]$.

The expression used to estimate the phase shift becomes zero for a neutrally buoyant bubble, and although it does not converge to proper limits for $\hat{f} \rightarrow 0$ or $\hat{f} \rightarrow \infty$, it matches the simulated data with reasonable accuracy within the tested range $\left(R^{2}>0.8\right.$ for most cases).

\subsection{The slip velocity around an airfoil leading edge}

The hypothesis set in the beginning of this chapter is tested by performing a numerical simulation in the potential flow (obtained using XFOIL, Drela 1989) around the leading edge of an airfoil at incidence $\alpha=14^{\circ}$. The airfoil is a section of a Fokker 100 aircraft wing (model 5-6 with retracted flap in Reinders W 1994) $67.59 \mathrm{~cm}$ chord, the model tested in the experiments of chapter 5 . The particles are released three chords upstream of the airfoil's leading edge, along a transverse line within $y / c=[-0.5,0.15]$ at every $0.01 \mathrm{c}$. Fifty particles are released per position, randomly distributed in space around the given reference points, for better resolving the spatial gradients. The Lagrangian simulations are performed for a $20 \%$ lighter-than-air bubble $(\hat{\rho}=0.8)$ of $0.5 \mathrm{~mm}$ diameter, $v=15 \mathrm{~mm}^{2} / \mathrm{s}$ and $U_{\infty}=70 \mathrm{~m} / \mathrm{s}$ (approaching experimental conditions). The diameter and chord Reynolds numbers are, respectively, $\operatorname{Re}_{d}=2.3 \times 10^{3}$ and $\operatorname{Re}_{c}=3.2 \times 10^{6}$, where $U_{\infty}$ is the reference velocity. 
Fig. 9 Comparison of slip velocity estimations with the numerical simulation results for a light particle $(\hat{\rho}=0.8)$ in the Eulerian frame of reference: a numerical simulation; b proposed approximation, Eqs. (20), (24) and (28); c approximation (adapted) from Mei (1996) in a rectilinear oscillating flow, Eq. (18); d no-slip acceleration assumption, Eq. (10). Notice that the colour scale is different in the latter case
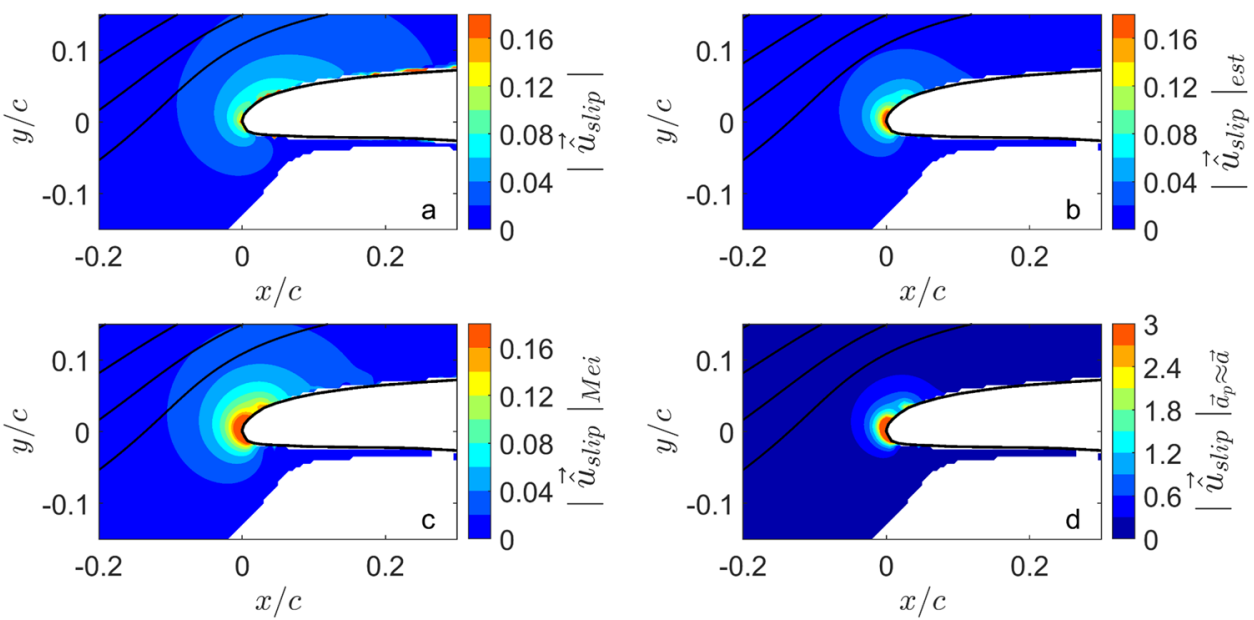
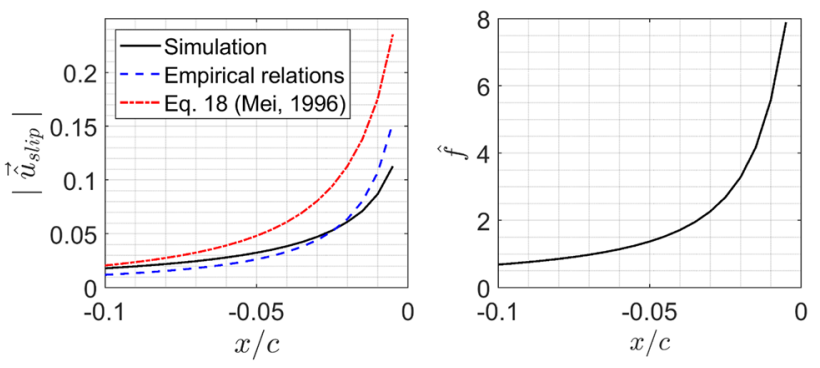

Fig. 10 Left: Comparison of slip velocity estimates along the line $y / c=0$, given by empirical relations obtained in this study, Eqs. (20), (24) and (28), and from Eq. (18), adapted from Mei (1996). Right: Increase in the normalized frequency as the particle approaches the model

The data are gridded using square bins of $0.5 \%$ chord length. Within each bin, the scattered velocity is fitted using a quadratic function in both spatial coordinates, following the approach discussed in Agüera et al. (2016). The mean velocity is taken as the fitted value at the centre of the bin. For convenience (as it should become clear when other angles of attack are included in the experimental part), the coordinate system in the Eulerian frame of reference is switched to the airfoil coordinates, where $x$ is the chordwise direction and $y$ is the normal to chord direction.

The slip velocity obtained from the numerical simulation is shown in Fig. 9a. In comparison, the slip velocity obtained from Eq. (20), with $\tilde{u}_{p}$ and $\beta_{s}$ obtained from Eqs. (24) and (28), is shown in Fig. 9b. The close agreement between the approximation and the simulated data supports the validity of the hypothesis that the flow around an object may be approximated by that of a complex sinusoid. The approximation given in Eq. (18) (Fig. 9c), based on Mei's work, overestimates the slip velocity at higher frequencies, as the particle approaches the model surface. Furthermore, estimating the slip velocity from Eq. (10), which requires negligible acceleration difference and neglects unsteady forces, leads to an overestimation of up to about $3000 \%$ of the simulated value (Fig. 9d).

A closer comparison of the empirical estimations herein developed with Mei's work is performed along the line $y / c=0$ in Fig. 10. The empirical expressions (24) and (28) give accurate estimations for $\hat{f}>1$, being within $20 \%$ of the simulated value for $1<\hat{f}<5$. The errors from the estimations given by Eq. (18) continuously increase as $\hat{f}$ increases, reaching about $100 \%$ of the actual $\left|\overrightarrow{\hat{u}}_{\text {slip }}\right|$ at $\hat{f}=5$. This is most likely because Eq. (18) does not account for drag corrections and, therefore, should become less accurate as $\operatorname{Re}_{p}$ increases.

\subsection{Density estimation}

Usually in a PIV experiment with HFSB, the bubble density is tuned using mass flow controllers set at appropriate flow rates that have been obtained through controlled experiments (Faleiros et al. 2019). However, it is good practice to verify this information during the measurements for an assessment of the errors. Hence, a procedure is defined to retrieve the bubbles' density from HFSB measurements for which a reference flow is available (e.g. DEHS measurements). The steps involved are outlined below by considering numerical simulations of HFSB tracers of given density in a reference potential flow solution around an airfoil.

A least square optimization is used to retrieve the particle density from Eqs. (20), (24) and (28) presented, by estimating the slip velocity for several density values within $\hat{\rho}=[0.5,1.5]$ and comparing with the numerical simulation for $\hat{\rho}=0.8$. The best match with the simulated particle velocity is obtained by minimizing the sum of squares: 
Fig. 11 Flow frequency (left) and phase angle (right)
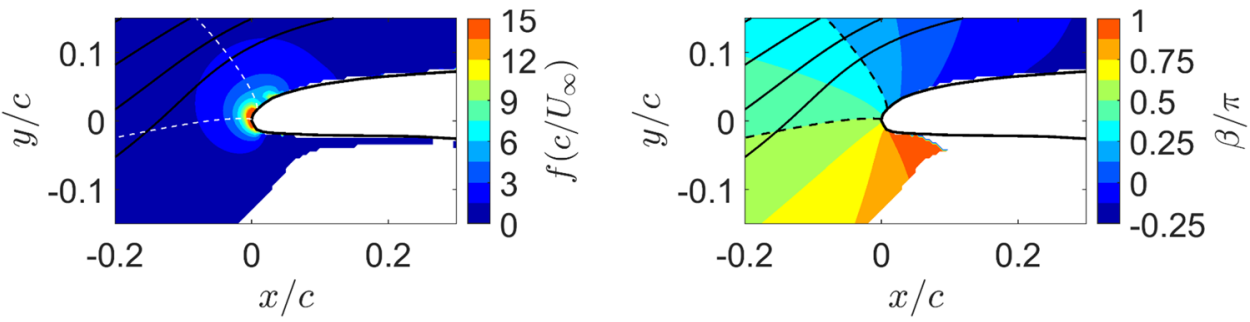

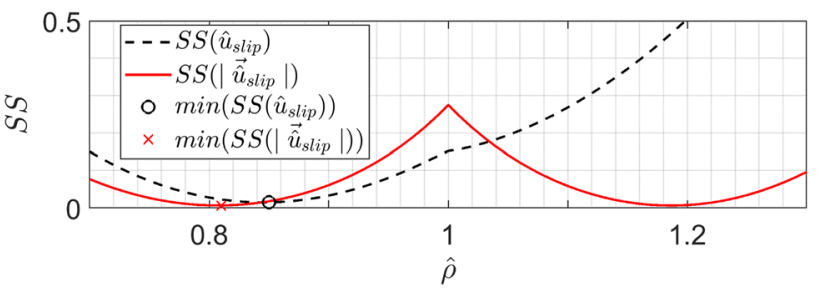

Fig. 12 Sum of squares of the difference between the slip velocity from the simulation $(\hat{\rho}=0.8)$ and the estimated slip velocity for $\hat{\rho}=[0.5,1.5]$. The local minimum of $\operatorname{SS}\left(\left|\overrightarrow{\hat{u}}_{\text {slip }}\right|\right)$ for $\hat{\rho}<1$ is found when $\hat{\rho}_{\text {est }}=0.81$

$\operatorname{SS}\left(\left|\overrightarrow{\hat{\mathrm{u}}}_{\text {slip }}\right|\right)=\sum\left(\left|\overrightarrow{\hat{\mathrm{u}}}_{\text {slip }}\right|-\left|\overrightarrow{\hat{\mathrm{u}}}_{\text {slip }}\right|_{\text {est }}\right)^{2}$,

where the subscript est stands for estimated value. This procedure is applied within $f c / U_{\infty}>3$ (Fig. 11, left) and $\pi / 4<\beta<\pi / 2$ (Fig. 11, right), which is deemed well modelled by the proposed approximation.

The $S S\left(\left|\overrightarrow{\hat{u}}_{\text {slip }}\right|\right)$ value has two local minimums (Fig. 12), one for $\hat{\rho}<1$ and another for $\hat{\rho}>1$. The correct local minimum is selected by considering the minimum sum of squares of the streamwise slip velocity, $S S\left(\hat{u}_{\text {slip }}\right)$. Although the density estimate from the latter is less accurate than given by $S S\left(\left|\overrightarrow{\hat{u}}_{\text {slip }}\right|\right)$, it evidently distinguishes between lighter- and heavier-than-air particles. From the minimum $S S\left(\hat{u}_{\text {slip }}\right)$, it is obtained that $\hat{\rho}<1$. The local minimum $S S\left(\left|\overrightarrow{\hat{u}}_{\text {slip }}\right|\right)$ in the lighter-than-air part yields an estimated density ratio of $\hat{\rho}_{\text {est }}=0.81$, only $1.25 \%$ larger than the simulated value. This result indicates that this method can be used for relatively accurate estimation of particle density from measurements of the slip velocity.

\section{Velocity fluctuations due to density dispersion}

The consequences of HFSB slip velocity dispersion, which was experimentally quantified (Morias et al. 2016; Faleiros et al. 2019) through the standard deviation of $\tau_{p}^{*}(\sim 40 \mu \mathrm{s})$, are herein considered.

\subsection{Sources of time response dispersion}

The two main particle parameters affecting the slip velocity are $\rho_{p}$ and $\mathrm{d}_{p}$, whose standard deviations $\sigma_{\rho}$ and $\sigma_{d}$, respectively, are the main sources of dispersion. The generation of HFSB in the bubbling regime is crucial to guarantee low-diameter dispersion. The coefficient of variation of the HFSB diameter $C V_{d}=\sigma_{d} / \mathrm{d}_{p}$ is $3 \%$ in the bubbling regime, but as large as $13 \%$ in the jetting regime (Faleiros et al. 2019). However, no correlation between diameter and time response dispersions has been observed, with measurements of $\sigma_{\tau}$ in both regimes being of the same order. This may be understood through Reynolds averaged decomposition of the time response as defined in Eq. (11), assuming $\phi\left(\operatorname{Re}_{p}\right) \sim 1$ for simplicity without losing generality. Notice that the measurements mentioned above have been performed in regions of low slip acceleration, validating this discussion. Neglecting second-order terms and assuming constant $\hat{\rho}$, it reads as:

$\tau_{p}^{*}+\tau_{p}^{*^{\prime}}=\frac{(\hat{\rho}-1)\left(\mathrm{d}_{p}^{2}+2 \mathrm{~d}_{p} \mathrm{~d}_{p}^{\prime}\right)}{18 v}$.

Subtracting $\tau_{p}^{*}$ from both sides, the time response dispersion is given as:

$\sigma_{\tau}=\sqrt{\left(\tau_{p}^{*^{\prime}}\right)^{2}}, \sigma_{\tau}=\frac{(\hat{\rho}-1) \mathrm{d}_{p}^{2}}{9 v} C V_{d}$.

Therefore, for a neutrally buoyant bubble $(\hat{\rho}=1)$ the diameter dispersion does not affect the time response dispersion. In fact, the diameter dispersion only affects $\sigma_{\tau}$ if there is a substantial deviation from the neutral buoyancy condition. Even if the mean density of half-millimetre bubble deviates $10 \%$ from the fluid density, $\sigma_{\tau}$ would still be $5 \mu$ s in the bubbling regime. In the worst case scenario, where jetting regime is present, then $\hat{\rho}=1.1$ results in $\sigma_{\tau} \approx 25 \mu \mathrm{s}$. This supports the experimental observations that HFSB diameter dispersion is not the main drive causing time response dispersion.

Bubble density dispersion may occur independently of bubble size through variations of the soap film thickness. Direct measurements of HFSB density have not been taken 
so far, only indirectly through measurement of bubble size and time response (Morias et al. 2016; Faleiros et al. 2019). Assuming exclusive density dispersion, the Reynolds average decomposition yields:

$\sigma_{\tau}=\frac{\hat{\rho} \mathrm{d}_{p}^{2}}{18 \nu} C V_{\rho}$,

where $C V_{\rho}=\sigma_{\rho} / \rho_{p}$ is the density coefficient of variation. Thus, for a distribution of bubbles with a mean density equal to that of the flow, constant diameter $\mathrm{d}_{p}=0.5 \mathrm{~mm}$ and time response dispersion of $\sigma_{\tau}=40 \mu$ s, the density coefficient of variation is $C V_{\rho}=0.043$ (in air at NTP).

This effect may be translated to soap film thickness variation, through mass conservation analysis. Assuming the volume of the soap film to be much smaller than that of helium, such that the bubble volume equals the helium volume, it is possible to estimate the bubble thickness as:

$t \approx \frac{\left(\rho_{p}-\rho_{\mathrm{He}}\right) \mathrm{d}_{p}}{6 \rho_{\text {soap }}}$.

This means that for $\mathrm{d}_{p}=0.5 \mathrm{~mm}, \rho_{p}=\rho_{\text {air }}$ and $\rho_{\text {soap }}=1124 \mathrm{~kg} / \mathrm{m}^{3}$, the soap film thickness is on average $77 \mathrm{~nm}$. Additionally, if the changes in thickness are exclusively responsible for the density dispersion, then the film thickness standard deviation $\sigma_{t}$ is about $3.5 \mathrm{~nm}$ for $C V_{\rho}=0.043$. Therefore, a film thickness coefficient of variation $\left(C V_{t}=\sigma_{t} / t\right)$ of only $5 \%$ is enough to result in $40 \mu$ s dispersion of the time response. Although this analysis remains to be verified, it is plausible to assume that the soap film thickness varies during the process of bubble formation with a coefficient of variation in the same order of magnitude as that measured for the bubble diameter.

\subsection{Velocity fluctuation estimation}

The time response dispersion results in velocity fluctuations that may be falsely interpreted as turbulence. In a two-component PIV measurement, the flow turbulence intensity,
$I=\sqrt{\frac{u_{\mathrm{rms}}^{2}+v_{\mathrm{rms}}^{2}}{2}}$,

is inferred from the root mean square (RMS) of the particle velocity fluctuation, whose streamwise component (and similarly the transverse component) reads as

$u_{p, \mathrm{rms}}=\sqrt{u_{p}^{\prime 2}}=\sqrt{E\left(u_{p}^{2}\right)-E\left(u_{p}\right)^{2}}, E\left(u_{p}\right)=\sum_{i=1}^{k} P_{i} u_{p, i}$,

where $u_{p}^{\prime}=u_{p}-\left\langle u_{p}\right\rangle, E$ is the expected value and $P$ is the probability of the outcome $u_{p}$. In steady flows, $u_{p, \text { rms }}$ may be rewritten as:

$u_{p, \text { rms }}=\sqrt{E\left(u_{\text {slip }}^{2}\right)-E\left(u_{\text {slip }}\right)^{2}}, E\left(u_{\text {slip }}\right)=\sum_{i=1}^{k} P_{i} u_{\text {slip }, i}$.

For an error assessment of this effect, the slip velocity may be estimated from Eq. (20), (24), (28), while the probability $P$ may be obtained by assuming that the slip velocity dispersion is exclusively resultant from the particle density distribution. Assuming a Gaussian distribution $N \sim\left(\rho_{p}, \sigma_{\rho}^{2}\right), P\left(\rho_{p}\right)$ is calculated as

$P\left(\rho_{p}\right) \approx F\left(\rho_{p}+\frac{\Delta \rho}{2}\right)-F\left(\rho_{p}-\frac{\Delta \rho}{2}\right)$,

where $F\left(\rho_{p}\right)$ is the cumulative distribution function:

$F\left(\rho_{p}\right)=\frac{1}{2}\left[1+\operatorname{erf}\left(\frac{\rho_{p}-\rho_{p}}{\sigma_{\rho} \sqrt{2}}\right)\right]$.

The accuracy of this approximation for estimating $u_{p, \mathrm{rms}}$ is demonstrated by repeating the simulation of Sect. 3.3 for a particle of normally distributed density with $\hat{\rho}=0.8$ and $C V_{\rho}=0.1$. As before, the data are gridded into bins by fitting the scattered data with a quadratic function. The velocity fluctuations are then obtained as the difference of the simulated particle velocity to the local fit value. This results in more accurate calculation of $u_{p, \text { rms }}$ in comparison with
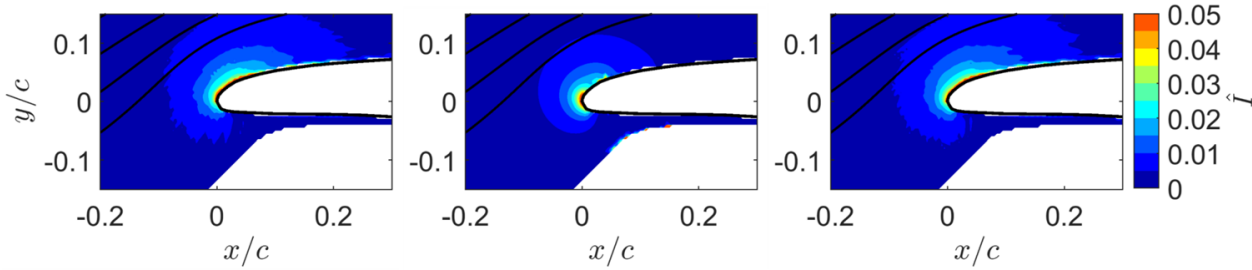

Fig. 13 Comparison of the velocity fluctuations for $\hat{\rho}=1$. Left: numerical simulation for $C V_{\rho}=0.1$ and $C V_{d}=0$. Middle: approximation from Eqs. (34) to (38) for $C V_{\rho}=0.1$ and $C V_{d}=0$. Right: numerical simulation for $C V_{\rho}=0.1$ and $C V_{d}=0.1$ 
simple subtraction of the bin ensemble average (Agüera et al. 2016).

The normalized turbulence intensity $\left(\hat{I}=I / U_{\infty}\right)$ around the airfoil leading edge obtained from the numerical simulation is shown in Fig. 13 (left). For the conditions tested, $I$ reaches about $3-4 \%$ of $U_{\infty}$ around the leading edge. There is a good agreement between the simulated $\hat{I}$ levels and that obtained from the proposed approximation (Fig. 13, middle), with the magnitude of the turbulence intensity error being well captured. Additionally, if a diameter coefficient of variation $C V_{d}$ of $10 \% d_{p}$-dispersion typical of jetting regimeis included in the simulations (Fig. 13, right), the results remain virtually unchanged. This supports the arguments given in the discussion of Sect. 4.1 that the influence of size dispersion on the slip velocity distribution is negligible in comparison with that of density dispersion.

\section{Experimental procedure, image processing and uncertainty}

The procedures developed in chapter 3 to estimate slip velocity and in chapter 4 to obtain velocity RMS from density dispersion are applied to evaluate the HFSB tracing fidelity in large-scale PIV measurements.

\subsection{Set-up of experiments}

The experiments are performed in the low-speed tunnel (LST) of the German-Dutch wind (DNW) tunnels, a closed-circuit tunnel with a closed test section of $3 \mathrm{~m}$ (height) $\times 2.25 \mathrm{~m}$ (width) cross-section, area contraction ratio of 9:1 and free stream turbulence level of approximately $0.03 \%$. The $2 \mathrm{D}$ high-lift airfoil represents an outer wing section of the Fokker 100 aircraft (model 5-6, Reinders W 1994) of scale 1:4.96 and chord of $67.59 \mathrm{~cm}$ and was tested with retracted flap. The airfoil was installed vertically spanning the test section height. The measurements were taken at 15,40 and $70 \mathrm{~m} / \mathrm{s}$ free stream velocity and at three angles of attack $\alpha=\left\{9^{\circ}, 14^{\circ}, 17^{\circ}\right\}$.

The planar two-component PIV system (Fig. 14) features two LaVision Imager sCMOS cameras $(2560 \times 2160$ $\mathrm{px}^{2}, 16$ bit, $6.5 \mu \mathrm{m}$ pixel pitch) equipped with $50 \mathrm{~mm}$ focal length objectives (lens aperture diameter of $\mathrm{f} / 16$ for HFSB and $\mathrm{f} / 5.6$ for DEHS). The cameras were installed on the top of the test section with their optical axis perpendicular to the laser sheet at a distance of about $1.5 \mathrm{~m}$, yielding an optical magnification of 0.03 , a digital imaging resolution of $0.2 \mathrm{~mm} / \mathrm{px}$ and a combined FoV of $0.95 \times 0.4 \mathrm{~m}^{2}$, covering the whole airfoil. A Quantel Evergreen $200 \mathrm{Nd}$ :YAG laser $(2 \times 200 \mathrm{~mJ} /$ pulse at $15 \mathrm{~Hz})$ was used for the particle illumination. The laser sheet thickness was $10 \mathrm{~mm}$ for HFSB and about $4 \mathrm{~mm}$ for DEHS. The laser power was set at $40 \%$ for the former and at $100 \%$ for the latter. As it can be observed, the imaging settings were at the limit in the case of DEHS, in terms of enhancing the optical signal, while the laser power had to be set to low power and the camera aperture fully closed for HFSB to avoid saturation. Thus, the volume achieved with HFSB could have been considerably larger. The acquisition and optical imaging conditions are summarized in Tables 2 and 3.

\subsection{HFSB generation}

The system of HFSB generation is composed of a fluid supply unit (FSU), fluid supply lines, flow resistors and bubble generators attached to the seeding rake. The in-house built FSU is composed of vessels and valves that can be operated remotely to pressurize and depressurize the fluid supply lines. Pressure flow controllers (coupled with mass flow meters) from Bronkhorst control the flow rates of helium, soap and air. Flow resistors guarantee equal mass
Fig. 14 Experimental set-up: top view (left) and side view (right). HFSB are generated in the settling chamber, minimizing flow intrusion. The seeding rake is positioned off centre, optimizing the bubble concentration in the measurement area. Dimensions in centimetres

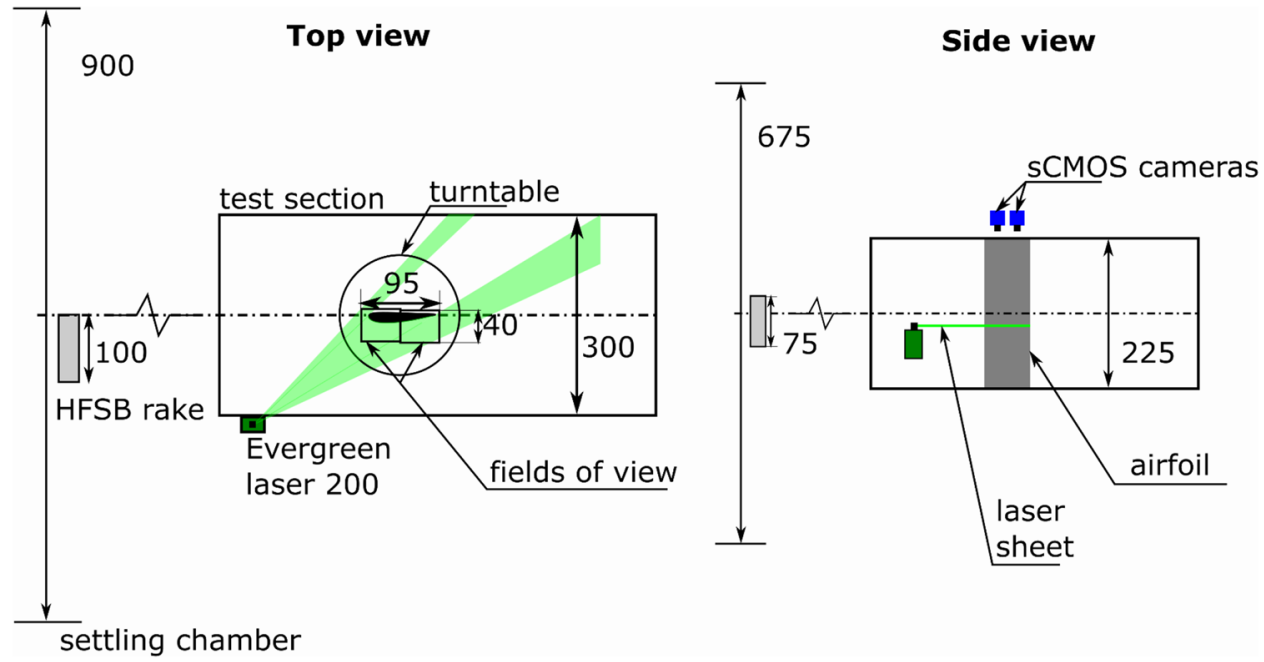


Table 2 Test matrix

\begin{tabular}{lllllll}
\hline$\alpha$ & $U_{\infty}(\mathrm{m} / \mathrm{s})$ & $\operatorname{Re}_{c}\left(10^{6}\right)$ & $\operatorname{Re}_{d}\left(10^{3}\right)$ & $\begin{array}{l}\text { Pulse separa- } \\
\text { tion }(\mu \mathrm{s})\end{array}$ & $\mathrm{N}^{\circ}$ images HFSB & $\begin{array}{l}\mathrm{N}^{\circ} \\
\text { images } \\
\text { DEHS }\end{array}$ \\
\hline $9^{\circ}$ & 15 & 0.7 & 0.5 & 105 & 2000 & 2000 \\
& 40 & 1.8 & 1.3 & 45 & 5500 & 6000 \\
& 70 & 3.2 & 2.3 & 30 & 9500 & 1000 \\
$14^{\circ}$ & 15 & 0.7 & 0.5 & 105 & 10,000 & 5000 \\
& 40 & 1.8 & 1.3 & 45 & 27,000 & 5000 \\
& 70 & 3.2 & 2.3 & 30 & 20,000 & 2000 \\
$17^{\circ}$ & 40 & 1.8 & 1.3 & 45 & 27,000 & 5000 \\
& 70 & 3.2 & 2.3 & 30 & 37,000 & 3000 \\
\hline
\end{tabular}

Table 3 Imaging conditions

\begin{tabular}{lll}
\hline Seeding & DEHS & HFSB \\
\hline Camera sensor size & $2560 \times 2160 \mathrm{px}^{2}$ & $2560 \times 2160 \mathrm{px}^{2}$ \\
Camera objective focal length & $50 \mathrm{~mm}$ & $50 \mathrm{~mm}$ \\
Combined field of view & $0.95 \times 0.40 \mathrm{~m}^{2}$ & $0.95 \times 0.40 \mathrm{~m}^{2}$ \\
Image resolution & $0.2 \mathrm{~mm} / \mathrm{px}$ & $0.2 \mathrm{~mm} / \mathrm{px}$ \\
Lens aperture diameter & $\mathrm{f} / 5.6$ & $\mathrm{f} / 16$ \\
Laser sheet thickness & $4 \mathrm{~mm}$ & $10 \mathrm{~mm}$ \\
Acquisition frequency & $15 \mathrm{~Hz}$ & $15 \mathrm{~Hz}$ \\
Laser pulse energy & $200 \mathrm{~mJ}$ & $80 \mathrm{~mJ}$ \\
\hline
\end{tabular}

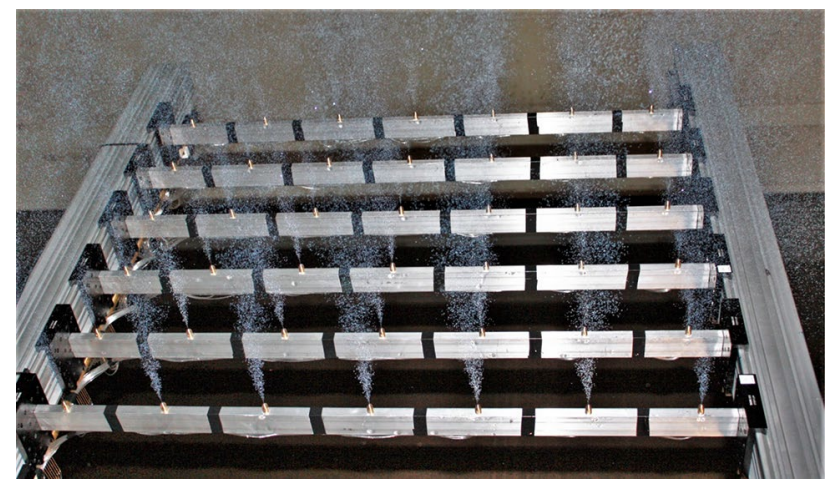

Fig. 15 NLR's seeding rake of 42 bubble generators

flow distribution to the bubble generators. These are CNCmanufactured nozzles of $1 \mathrm{~mm}$ orifice diameter (Faleiros et al. 2019) designed by the Royal Netherlands Aerospace Centre (NLR). The bubble generator dimensions, working principle, regimes of generation and bubble properties have been recently studied by the authors (Faleiros et al. 2019). In the present experiments, the average volume flow rates per generator were $80 \mathrm{l} / \mathrm{h}$ of air, $9.5 \mathrm{l} / \mathrm{h}$ of helium and $9.5 \mathrm{ml} / \mathrm{h}$ of soap, yielding 30,000 bubbles/s (per generator) of nominal bubble density of $1.1 \mathrm{~kg} / \mathrm{m}^{3}(\hat{\rho} \sim 0.9)$ and mean diameter of $0.5 \mathrm{~mm}$. The seeding rake, an array composed of six
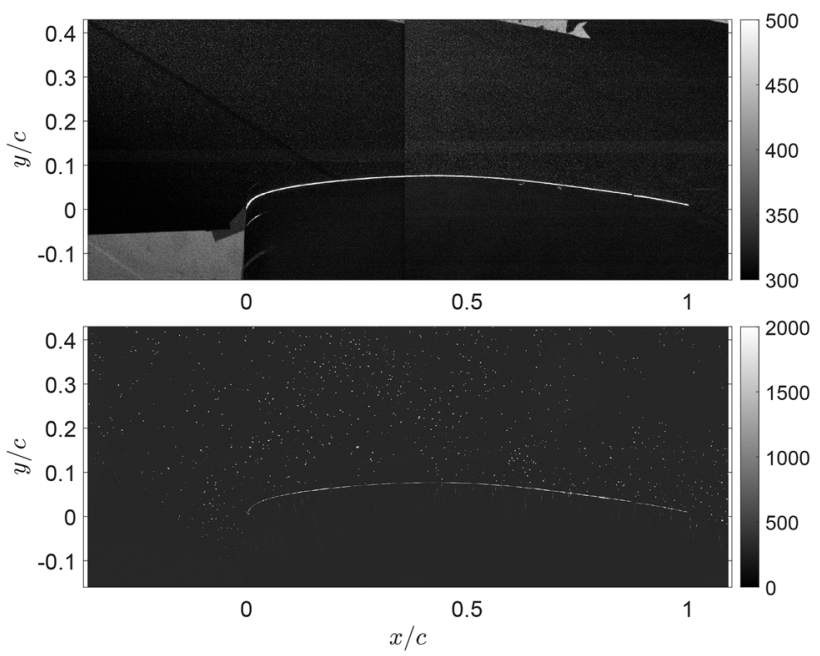

Fig. 16 Raw images obtained with DEHS (top) and HFSB (bottom)

horizontal segments, where 42 bubble generators are distributed in intervals of $15 \mathrm{~cm}$ (Fig. 15), was installed in the settling chamber. Its influence on the flow was not quantified. However, the system was also in place during the DEHS measurements, with equivalent air flows through the bubble generator nozzles, to mitigate its influence on slip velocity measurements. The bubble system provides a seeded stream tube of about of $0.75 \mathrm{~m}$ (height) $\times 0.90 \mathrm{~m}$ (width) crosssection area, with an injection rate of approximately 1.3 million bubbles/s. The resultant stream tube of HFSB after the wind tunnel contraction is $0.25 \mathrm{~m}$ (height) $\times 0.30 \mathrm{~m}$ (width). The bubble concentration in the test section is 0.24 bubble/ $\mathrm{cm}^{3}$ for $U_{\infty}=70 \mathrm{~m} / \mathrm{s}, 0.42$ bubble $/ \mathrm{cm}^{3}$ for $U_{\infty}=40 \mathrm{~m} / \mathrm{s}$ and 1.1 bubbles $/ \mathrm{cm}^{3}$ for $U_{\infty}=15 \mathrm{~m} / \mathrm{s}$. This concentration level is not sufficient to perform spatial correlation analysis of the instantaneous images as it requires typically $5-10$ particles per interrogation window, for which it would be necessary an interrogation volume of at least $5 \mathrm{~cm}^{3}$ at the lowest speed, resulting in low spatial resolution. 


\subsection{Data quality}

The raw images obtained with HFSB (Fig. 16, bottom) and DEHS (Fig. 16, top) elucidate the advantage of both particles, while DEHS measurements benefit from higher particle concentrations, HFSB are brighter, providing two orders of magnitude larger signal-to-noise ratios (SNR). The signal from DEHS particles is of the same order of magnitude of the noise level (300 counts), reaching an SNR of about 1.5. HFSB particles reach over 30,000 counts with SNR $>100$. It is also noted a chordwise light intensity gradient (decreasing in intensity from left to right), observed in the DEHS raw images of both cameras. The light intensity scattered by particles upstream of the camera axis is higher than that scattered by particles downstream of it, due to a small (but significant) component of forward scattering (Raffel et al. 2018) from the former-there is a $20^{\circ}$ angle between the light rays reaching the camera from the most upstream to the most downstream positions. The DEHS tracer signal is, therefore, lower near the downstream edges of the fields of view, compromising the DEHS data quality in these regions.

\subsection{Image processing}

The data obtained with DEHS were processed using the crosscorrelation algorithm from the LaVision software DaVis 10. The final interrogation window used is $48 \times 48$ pixels large $(0.96 \mathrm{~cm} \times 0.96 \mathrm{~cm}$ in physical space). With an overlap of 75\% among adjacent interrogation windows, the vector spacing is $0.24 \mathrm{~cm}$. Vectors whose absolute difference from the mean exceeded two standard deviations were excluded prior to obtaining statistics of the first and second moments of velocity.

The data acquired with HFSB were processed using an inhouse algorithm developed with MATLAB. Particles were identified based on local maxima, their centre was obtained using a 3-point Gaussian fit (Raffel et al. 2018), which was used for pairing to the particle positions in the next frame according to the nearest neighbour criterion. The particle displacement ( $2 \mathrm{~mm}$ in the free stream) was sufficiently small compared to the average particle distance (about $20 \mathrm{~mm}$ at $40 \mathrm{~m} / \mathrm{s}$ ). The FoV was then gridded into square bins of $1.5 \mathrm{~cm}^{2}$ for statistical analysis. The velocity moments were obtained in the same manner as described in chapters 3 and 4 .

\subsection{Uncertainty quantification}

The uncertainty on the measured velocity with PIV is dominated by the uncertainty on the measured particle displacement, which is typically $0.1 \mathrm{px}$ (Raffel et al. 2018). The particle displacements during the experiments were about $10 \mathrm{px}$, yielding a measurement error on the instantaneous velocity of $1 \%$. Furthermore, assuming a Gaussian distribution for the random error, the expanded uncertainty estimate of the mean

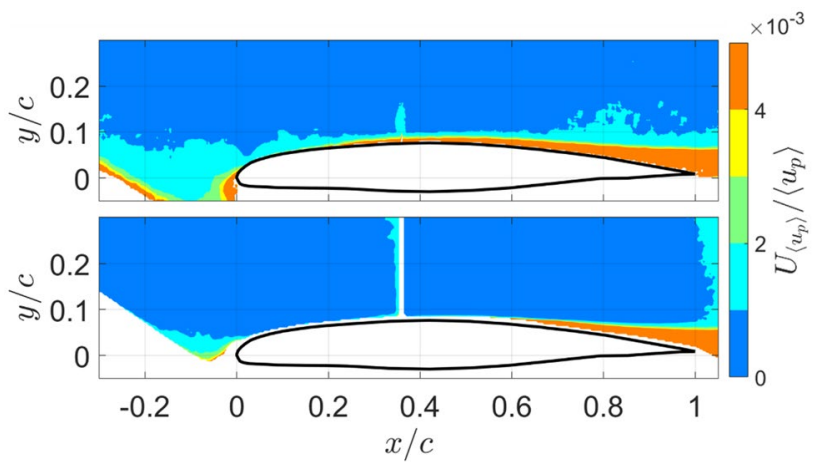

Fig. 17 Uncertainty on the streamwise velocity component for $U_{\infty}=40 \mathrm{~m} / \mathrm{s}$ and $\alpha=14^{\circ}$

streamwise velocity $u$ (analogously for the $v$ component), respectively, with a 95\% confidence level is given as (Benedict and Gould 1996; Sciacchitano and Wieneke 2016):

$U_{u}=1.96 \frac{\sigma_{u}}{\sqrt{N(x, y)}}$,

where $N(x, y)$ is the number of uncorrelated vectors at the grid $(x, y)$ location and $\sigma_{u}$ is the streamwise velocity standard deviation, which contains both the true velocity fluctuations and measurement errors. The uncertainty on the streamwise velocity component is almost everywhere about $0.1-0.2 \%$ of the local mean velocity (Fig. 17) with the exception of the separated region. Higher uncertainty at the leading edge region is observed for HFSB because of density dispersion. In addition, because the DEHS SNR is low, the uncertainty is also higher at the leading edge region, where the laser illumination is less intense, and a more restrictive mask was applied.

\subsubsection{Uncertainty on the identification of HFSB particle centre}

The HFSB glare point image is diffraction dominated (Appendix A1), which is well approximated by a Gaussian function (Adrian and Yao, 1985). The standard deviation of the Gaussian function that approximates the Airy disc is given as (Zhang et al. 2007):

$\sigma \approx 0.42 f_{\#} \lambda$,

where $\lambda$ is the laser light wavelength. This yields $\sigma=0.55$ px for $f_{\#}=16, \lambda=532 \mathrm{~nm}$ and camera pixel pitch of $6.5 \mu \mathrm{m}$. The glare point size and the distance between the glare points are estimated to be 3.3 and $1.7 \mathrm{px}$, respectively (see Appendix A1). This means that the glare points partially merge, yielding a single particle image. The peak intensity of the glare point $I_{\text {peak }}$ reflected on the external surface of the bubble is on average 1.34 larger than that reflected on the 
Fig. 18 Uncertainty on the particle centre identification. Left: Representation of the particle image along the illumination direction, based on estimated glare point size, distance the ratio of glare point intensities and Gaussian approximation to the Airy disc. Right: Particle images obtained experimentally between the glare points, mean

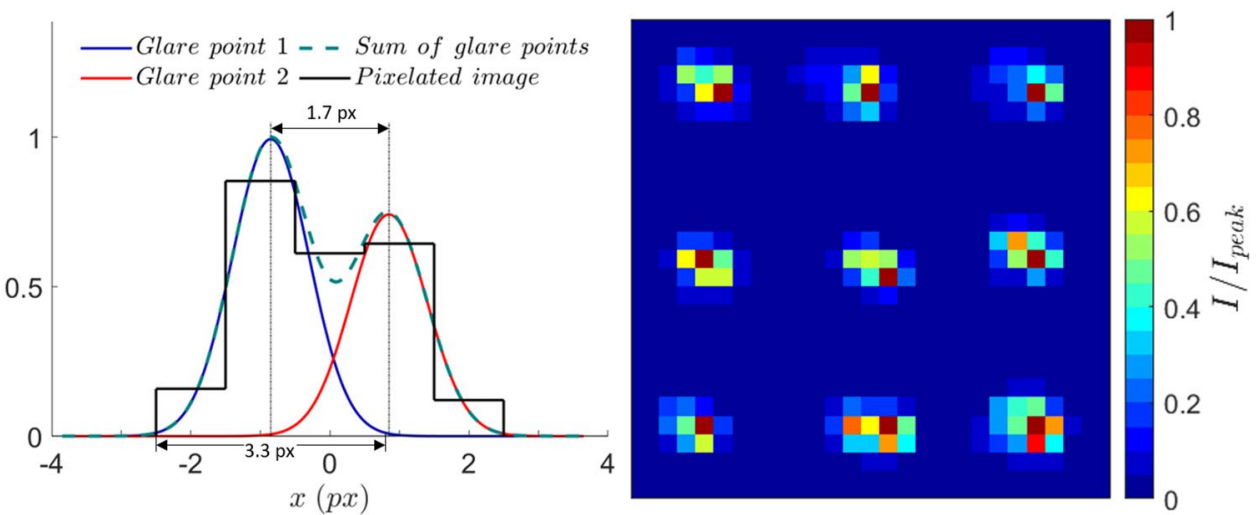

C

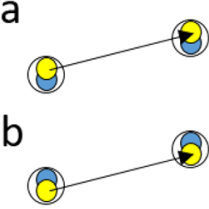

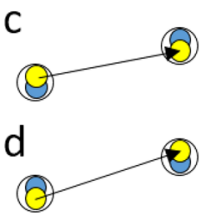

Fig. 19 Uncertainty on the displacement of HFSB with partially merged (on the image plane) glare points. In this sketch, the top and bottom dots represent the externally and internally reflected glare points, respectively (see Appendix 1). Sketches a. and b. (68\% of the cases) show a stable situation where the brightest glare point (yellow dot) remains the same in both frames. Sketches c. and d. (38\% of the cases) represent the situations in which the brightest glare point on the 2 nd frame is different from that on the 1 st frame

internal part (Appendix 2) for HFSB, which leads to a local maxima at the centre of the brightest glare point. A sketch of the particle image considering these characteristics is shown in Fig. 18 (left). Measured particle images intensities within a window of $9 \times 9$ pixels around the local maxima (Fig. 18, right) support the proposed representation. Estimation of the particle centre at the local maxima leads to a position uncertainty of about $\mathrm{d}_{g} / 2$, where $\mathrm{d}_{g}=\mathrm{d}_{p} / \sqrt{2}$ is the distance between the glare points. This is not concerning, as flow velocity changes should be negligible at the particle scale. The measured displacement is not affected by this effect, as long as the brightest glare point remains the same in both frames (Fig. 19a and b). However, analysis of data from previous measurements (Faleiros et al. 2018), where the glare point images are distinguishable from each other, seems to indicate that this is only the case in $62 \%$ of the measured data (Appendix 2), while in $38 \%$ of the cases the uncertainty is of the instantaneous particle displacement is in the order of the glare point distance ( $1.7 \mathrm{px}$ in this case). Taking this into account, the uncertainty on the mean particle displacement is estimated to be $0.7 \mathrm{px}\left(\sigma_{\Delta x}=0.62(0.1)+0.38(1.7)\right)$. With the illumination at about $45^{\circ}$ angle with the flow direction (Fig. 14), it yields an uncertainty of about $0.5 \mathrm{px}$ in the $u$ and $v$ velocity components. Fortunately, as shown in Fig. 19, cases $c$ and $d$ counteract each other, and the mean velocity is not affected significantly. This type of uncertainty mainly affects the velocity fluctuations and should be detected anywhere in the flow. As it is shown in Sect. 6.2, the turbulence intensity measured with HFSB in the free stream is comparable to that measured with DEHS. Therefore, this type of uncertainty has not affected the results significantly.

\section{Experimental results}

\subsection{Slip velocity}

The magnitude of the DEHS and HFSB velocities are shown in Fig. 20 for $U_{\infty}=40 \mathrm{~m} / \mathrm{s}$ and $\alpha=14^{\circ}$. The colour contours indicate a good agreement between both measurements. The overall agreement between the two measurements is verified by overlaying the HFSB velocity data with the DEHS velocity isolines (Fig. 21). Two measurement conditions are presented for the sake of conciseness: $40 \mathrm{~m} / \mathrm{s}$ at $\alpha=14^{\circ}$ and $70 \mathrm{~m} / \mathrm{s}$ at $\alpha=17^{\circ}$. The results show a good agreement for the two velocity components in both flow conditions, although with larger deviations at $70 \mathrm{~m} / \mathrm{s}$ and $17^{\circ}$ incidence.
Fig. 20 Velocity magnitude for $U_{\infty}=40 \mathrm{~m} / \mathrm{s}$ and $\alpha=14^{\circ}$, DEHS (left) and HFSB (right). Showing 1 every $9 \times 9$ vectors
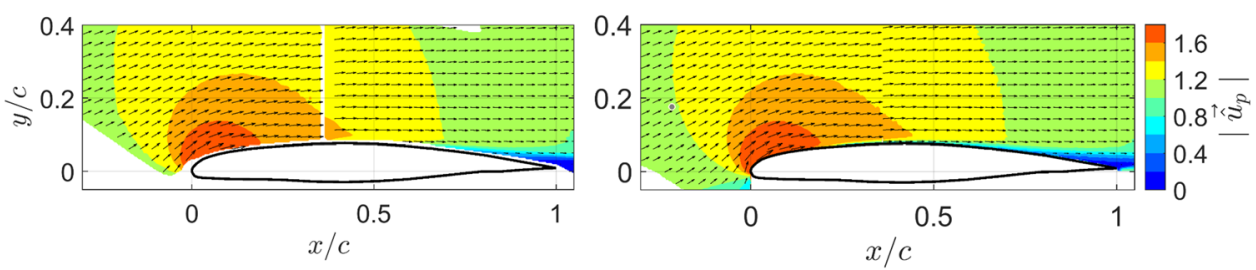
Overall, the slip velocity remains within $2 \%$ of $U_{\infty}$ (Fig. 22), with the exception of the leading edge and separated regions. In most cases, the slip velocity in the leading edge region is essentially within 4 to $6 \%$, reaching up to $12 \%$ of $U_{\infty}$ near the surface. In the most extreme case $(70 \mathrm{~m} / \mathrm{s}$ and $17^{\circ}$ ), the slip velocity approaching the surface near the leading edge reaches up to $20 \%$ of $U_{\infty}$. It is noted that the slip velocity in the separated region reaches about $10 \%$ of $U_{\infty}$ in some cases, in contradiction to previous results (Bourgoin et al. 2011; Faleiros et al. 2018). Close inspection of the data shows that the separation point shifted slightly in some cases between HFSB and DEHS, which could explain these differences. As the separation point has not been fixed through the use of a tripping device, a precise comparison of the slip velocity in the separated region is not possible.

The HFSB density is estimated through the least square optimization described in Sect. 3.4 (Table 4). A trend is observed which shows bubble density decreasing with wind tunnel speed. The bubbles are about $20-30 \%$ heavier than air at $15 \mathrm{~m} / \mathrm{s}$, within $10 \%$ difference from the neutral buoyancy condition at $40 \mathrm{~m} / \mathrm{s}$ and about $20 \%$ lighter than air at $70 \mathrm{~m} / \mathrm{s}$. This systematic density variation with wind tunnel speed is not explained by the generation process. Changes in the tunnel total pressure do not affect the mass flow rate of helium, which is mass flow controlled. The pressure-controlled soap input of about 4 bars renders tunnel total pressure variations negligible $(<1 \%$ of the input pressure). Even though the viscosity of the soap film is sensitive to temperature changes, causing variations in the volume flow rate, those were monitored with a mass flow meter and counteracted by readjusting the helium mass flow for a constant helium-to-soap flow rate ratio $\left(Q_{\mathrm{He}} / Q_{\text {soap }} \sim 1000\right)$. An alternative explanation is that the HFSB density changed after generation, due to soap film evaporation and diffusion of helium and air through the soap film. Both the tunnel temperature (Table 4) and the bubble residence time (the time from generation until the measurement) influence these physical processes. Shrinking of HFSB, as a result of helium diffusion, and a colour shift from red to blue, attributed to soap film thinning, have both been observed by Huhn et al. (2017), while studying
Fig. 21 Velocity contours of chordwise (top) and normal to chord (bottom) components. Left: $U_{\infty}=40 \mathrm{~m} / \mathrm{s}$ and $\alpha=14^{\circ}$. Right: $U_{\infty}=70 \mathrm{~m} / \mathrm{s}$ and $\alpha=17^{\circ}$. HFSB: colourfilled contours and dashed lines. DEHS: solid lines

Fig. 22 HFSB slip velocity with respect to DEHS measurements

Table 4 Density ratio $\hat{\rho}$ estimate per measurement condition
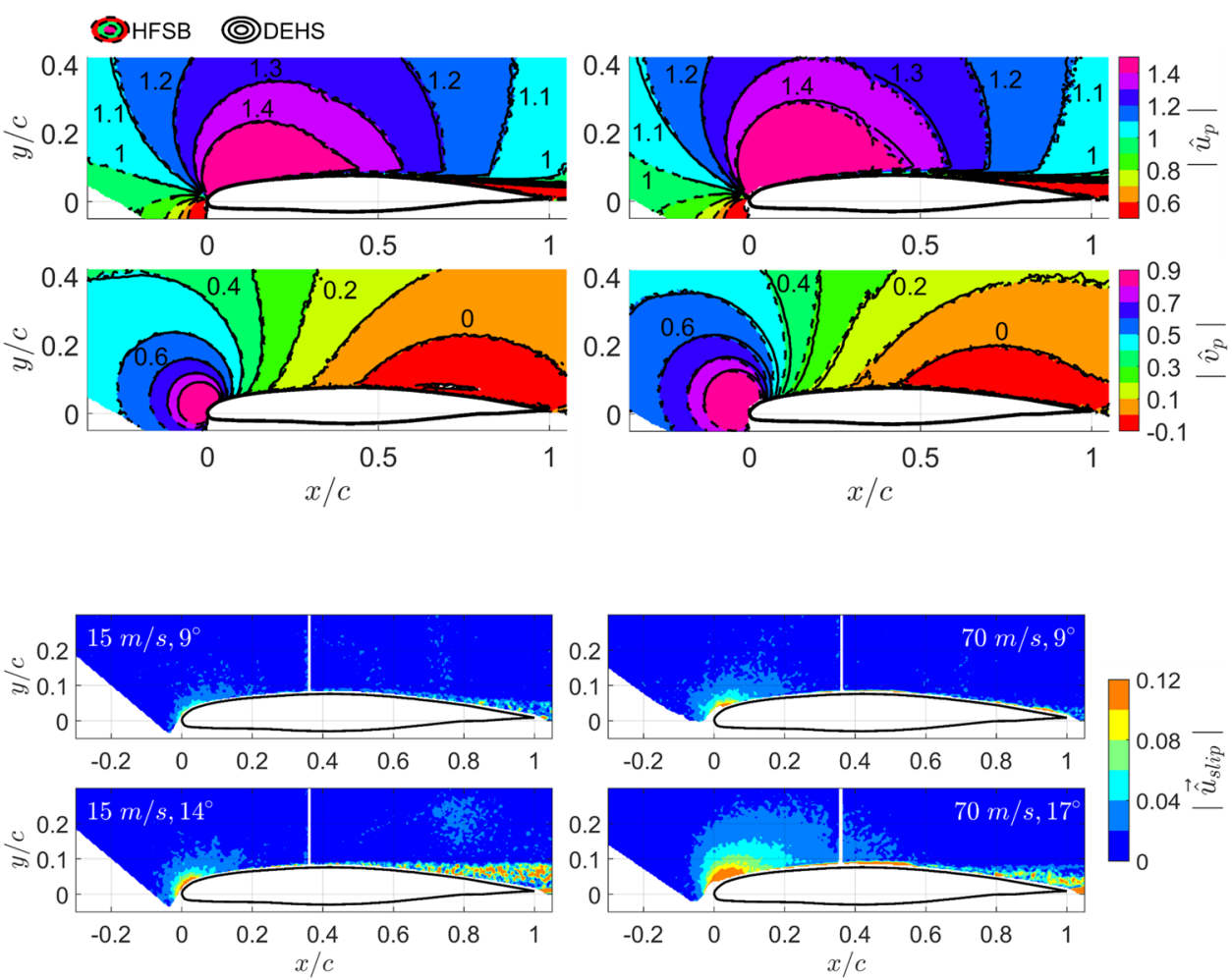

\begin{tabular}{llllllll}
\hline & \multicolumn{3}{l}{ Density ratio $\hat{\rho}$} & & & \multicolumn{3}{l}{ Total temperature $\left({ }^{\circ} \mathrm{C}\right)$} \\
\cline { 2 - 3 } & $15 \mathrm{~m} / \mathrm{s}$ & $40 \mathrm{~m} / \mathrm{s}$ & $70 \mathrm{~m} / \mathrm{s}$ & & $15 \mathrm{~m} / \mathrm{s}$ & $40 \mathrm{~m} / \mathrm{s}$ & $70 \mathrm{~m} / \mathrm{s}$ \\
\hline $9^{\circ}$ & 1.22 & 0.94 & 0.81 & & 24.7 & 25.2 & 28.6 \\
$14^{\circ}$ & 1.32 & 1.12 & 0.81 & & 31.1 & 14.6 & 33.7 \\
$17^{\circ}$ & - & 0.90 & 0.87 & & - & 25.6 & 27.6 \\
\hline
\end{tabular}


the HFSB lifetime. It is beyond the scope of this article to validate these conjectures.

From the HFSB density values, correspondent slip velocity fields are estimated (Fig. 23, bottom), based on the velocity fields measured with HFSB and Eqs. (20), (24) and (28). Those are compared to that of the measured slip velocity (Fig. 23, top). Despite measurement noise, there is sufficient agreement to support the validity of the density estimation procedure.

Fig. 23 Top: Slip velocity (velocity difference between HFSB and DEHS) measured experimentally at $9^{\circ}$ angle of attack for (from left to right) $U_{\infty}=\{15,40,70\} \mathrm{m} / \mathrm{s}$. Bottom: Slip velocity estimated from HFSB measurements, Eqs. (20), (24) and (28), and estimated density from least square optimization (Sect. 3.4)

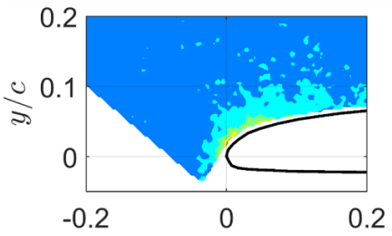

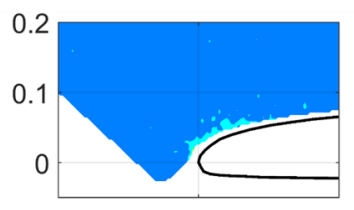
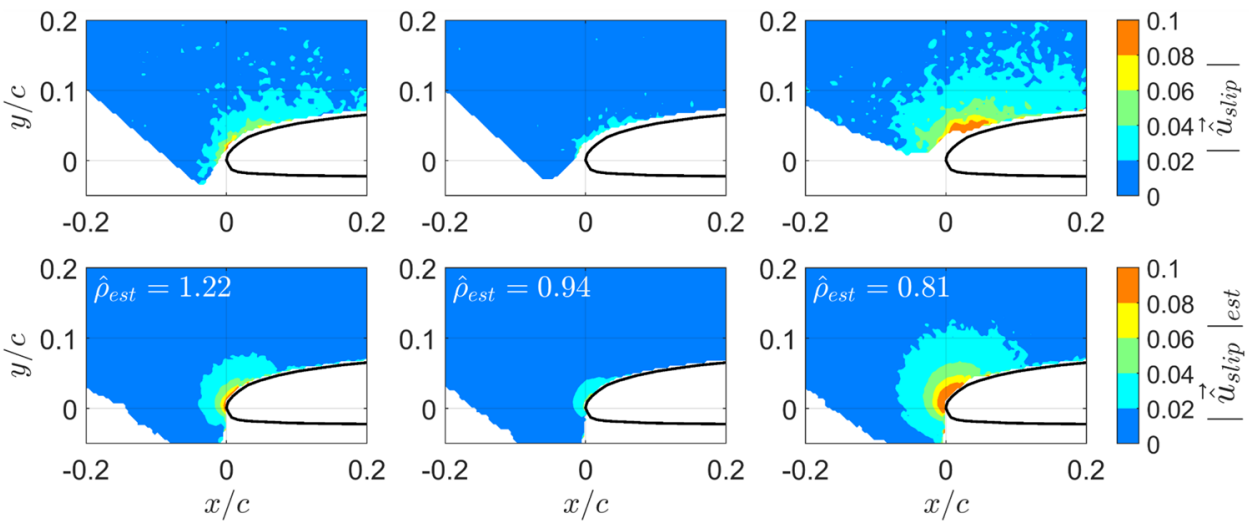

\begin{tabular}{|c|c|c|c|c|c|c|}
\hline & \multicolumn{3}{|l|}{ DEHS } & \multicolumn{3}{|l|}{ HFSB } \\
\hline & $15 \mathrm{~m} / \mathrm{s}$ & $40 \mathrm{~m} / \mathrm{s}$ & $70 \mathrm{~m} / \mathrm{s}$ & $15 \mathrm{~m} / \mathrm{s}$ & $40 \mathrm{~m} / \mathrm{s}$ & $70 \mathrm{~m} / \mathrm{s}$ \\
\hline $9^{\circ}$ & $2.0 \%$ & $1.4 \%$ & $1.7 \%$ & $1.9 \%$ & $1.9 \%$ & $2.2 \%$ \\
\hline $14^{\circ}$ & $1.7 \%$ & $1.6 \%$ & $1.6 \%$ & $1.7 \%$ & $1.8 \%$ & $2.1 \%$ \\
\hline $17^{\circ}$ & - & $1.8 \%$ & $1.6 \%$ & - & $2.3 \%$ & $2.3 \%$ \\
\hline
\end{tabular}

Fig. 24 Comparison between the velocity fluctuations measured with DEHS (left) and

HFSB (right)

Table 5 Normalized free stream turbulence intensity $\hat{I}_{\infty}$, taken as the spatial average of $I$ within a square of 0.05 chord side length with centre at $(x / c, y / c)=(-0.2$, 0.3 )
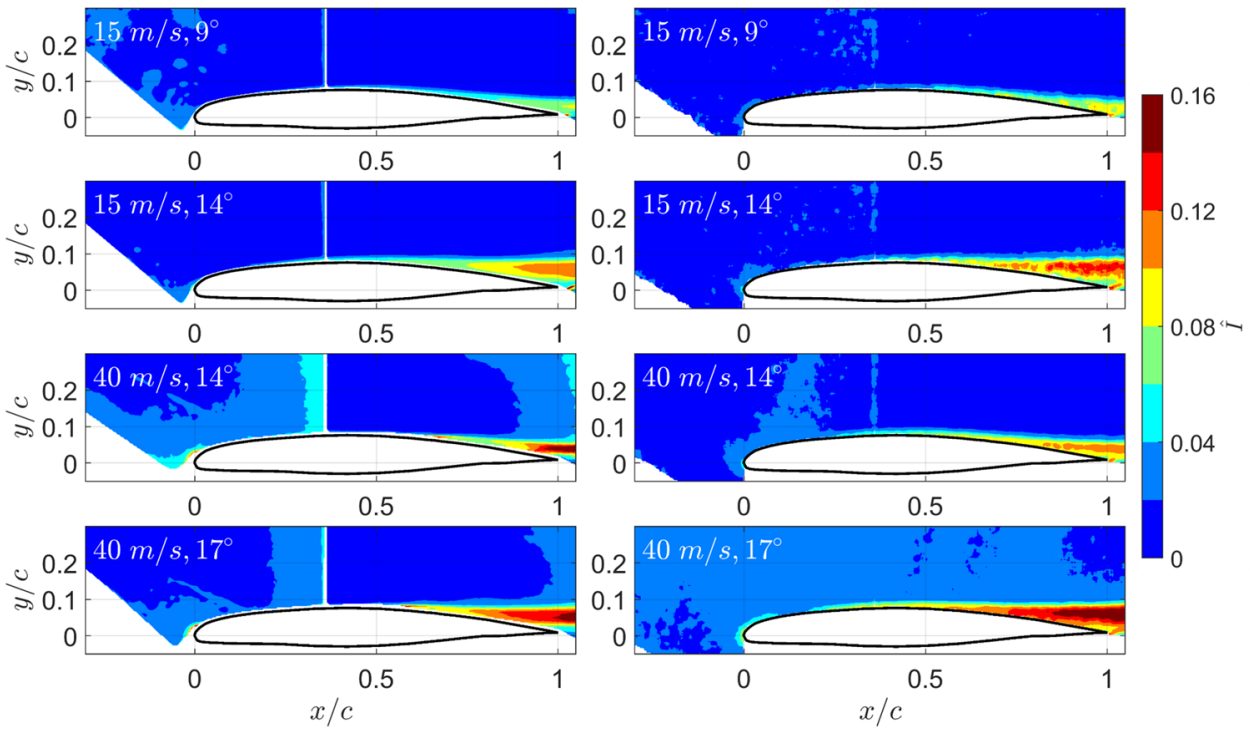
In general, a good qualitative agreement between DEHS and HFSB velocity fluctuations is observed (Fig. 24). The separated regions have similar topology, with HFSB measurements of turbulence intensity peak levels exceeding the reference by about $2 \%$, with the exception of the $40 \mathrm{~m} / \mathrm{s}$ and $14^{\circ}$ incidence case, where DEHS measurements overestimate the turbulence intensity levels close to the trailing edge. The light intensity gradient of DEHS images (Fig. 16) results in higher turbulence intensity in the downstream edges, where the SNR is lower, and is especially noticeable in the measurements at $40 \mathrm{~m} / \mathrm{s}$. The turbulence levels measured with HFSB close to the leading edge are between 2 and $4 \%$ of $U_{\infty}$ in most cases, except for $\alpha=17^{\circ}$, where it reaches $6 \%$. As discussed in chapter 4 , velocity fluctuations in the leading edge region are attributed to bubble density dispersion. It is noted, however, that no significant changes in the velocity fluctuations have been observed between the simulations at $14^{\circ}$ and $17^{\circ}$ incidence (not shown), posing the question of whether the leading edge fluctuations measured at $17^{\circ}$ are indeed exclusively due to bubble density variation or a combination of the latter with existent flow fluctuations that are known to occur at the leading edge in the onset of stall (e.g. Benton and Visbal, 2018).

The density coefficient of variation $C V_{\rho}$ is estimated via a least square optimization analogous to the one performed for the mean density estimation. The normalized density dispersion is mostly within 10 and $15 \%$ of the mean density (Table 6), with the exception of the data for $17^{\circ}$ incidence, where $\sigma_{\rho}$ is about $20 \%$ of the mean density. The density dispersion at $17^{\circ}$ might be overestimated, however, as mentioned above, due to possible extra flow fluctuations that occur in the onset of stall. Overall, the estimated values are at

Table 6 Density coefficient of variation $C V_{\rho}$ estimated per measurement condition

Fig. 25 Top: turbulence intensity levels measured experimentally for, from left to right, $15 \mathrm{~m} / \mathrm{s}$ and $9^{\circ}, 40 \mathrm{~m} / \mathrm{s}$ and $14^{\circ}$, and $40 \mathrm{~m} / \mathrm{s}$ and $17^{\circ}$, respectively. Bottom: the correspondent turbulence intensity estimated from Eqs. (34) to (38), with slip velocity obtained from Eqs. (20), (24) and (28), and estimated $C V_{\rho}$ least twice as large as those estimated in Sect. 4.1. The larger values might be explained by the extra uncertainties resultant from the simultaneous operation of a multi-nozzle system.

The $\hat{I}$ values measured with HFSB in the leading edge region (Fig. 25, top) are compared to $\hat{I}_{\text {est }}$ (Fig. 25, bottom), obtained using Eq. (34) to (38) and the estimated $C V_{\rho}$ (Table 6). Within the limitations of measurement and processing errors, and of statistical convergence, the estimated values show a good agreement with the experimental data, supporting the validity of the method proposed for estimation of HFSB density dispersion. Furthermore, the extra turbulence intensity caused by HFSB density dispersion is shown to be a localized effect, only occurring within regions of high flow frequency.

\section{Summary and conclusions}

This study has contributed to a more systematic assessment and prediction of the slip velocity of nearly neutrally buoyant tracers in typical conditions expected for large-scale PIV measurements in wind tunnels. It was shown that the slip velocity is governed by three non-dimensional parameters (as opposed to a single Stokes number), namely the ratio of particle to fluid density $\hat{\rho}$, the diameter-based Reynolds number $\operatorname{Re}_{d}$ and the local frequency $\hat{f}$ (normalized with the viscous timescale $d_{p}^{2} / v$ ).

An analogy has been made between the particle motion in a sinusoid flow and that of a particle travelling around an object, which provided generalization to the analysis of particle slip velocity in external aerodynamics. Empirical relations have been obtained through numerical simulations, allowing the estimation of the slip velocity without the need of time-consuming computations. A direct comparison to other available methods in the literature has shown significant improvement in the accuracy of the estimations, emphasizing the importance of considering unsteady forces and drag correction for the slip velocity estimation. The empirical relations obtained may be used to establish the limits of

\begin{tabular}{lccc}
\hline & $15 \mathrm{~m} / \mathrm{s}$ & $40 \mathrm{~m} / \mathrm{s}$ & $70 \mathrm{~m} / \mathrm{s}$ \\
\hline $9^{\circ}$ & 0.12 & 0.15 & 0.12 \\
$14^{\circ}$ & 0.10 & 0.10 & 0.11 \\
$17^{\circ}$ & - & 0.18 & 0.21 \\
\hline
\end{tabular}
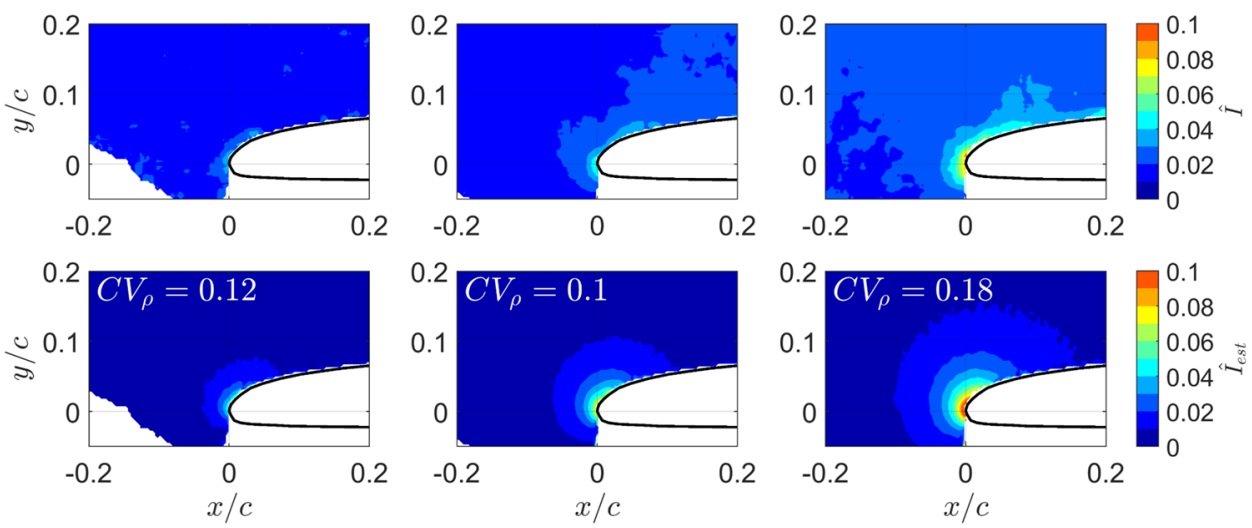
Table 7 Main equations used for the slip velocity analysis

\begin{tabular}{ll}
\hline & Equations \\
\hline Frequency and phase & $(19)$ \\
Slip velocity & $(20),(24)$ and (28) \\
Amplitude modulation & $(24)$ \\
Phase shift & $(28)$ \\
Slip velocity fluctuations & $(36)$ to (38) \\
\hline
\end{tabular}

the HFSB tracing fidelity, which are visualized by maps that relate the slip velocity to the non-dimensional parameters $\hat{\rho}, \operatorname{Re}_{d}$ and $\hat{f}$ (see Fig. 7 for the amplitude modulation). A summary of the main equations used for the slip velocity analysis is given in Table 7 .

The tracing fidelity of nominally $10 \%$ lighter-than-air HFSB is also verified through PIV experiments in the flow around an airfoil of $70 \mathrm{~cm}$ chord in typical conditions of subsonic wind tunnel measurements in aeronautics at 15, 40 and $70 \mathrm{~m} / \mathrm{s}$. The HFSB slip velocity was shown to be overall below $2 \%$ of $U_{\infty}$, with the exception of the high-acceleration region around the leading edge, where in most cases it is contained below $5 \%$ of $U_{\infty}$. Only in the most extreme measurement conditions, as in the onset of stall at $70 \mathrm{~m} / \mathrm{s}$, that the slip velocity close to the airfoil surface has reached values in the order of $10 \%$ of $U_{\infty}$. In general, the velocity errors were localized and had minor effects on the overall measurement quality.

The method has also been extended to allow evaluation of bubble density as a function of the slip velocity and of bubble density dispersion, through measurements of velocity fluctuations. Comparison of the experimental results with those from the numerical simulations indicate that the HFSB density has changed post-generation-remaining within $10-30 \%$ of the neutral buoyancy condition-most likely because of variations of wind tunnel temperatures and bubble residence time, which affect the density through evaporation of the soap film and helium diffusion. In addition, the HFSB density coefficient of variation with respect to the mean density was quantified to be approximately $10 \%$, resulting in measurement errors of turbulence intensity around the leading edge of up to $5 \%$ of $U_{\infty}$ for most test conditions.

\section{Appendix 1}

\section{HFSB glare point size, distance and merging}

When soap bubbles floating in the air are illuminated, the reflected light rays form two glare points on the image sensor of the recording device, resultant from reflections occurring

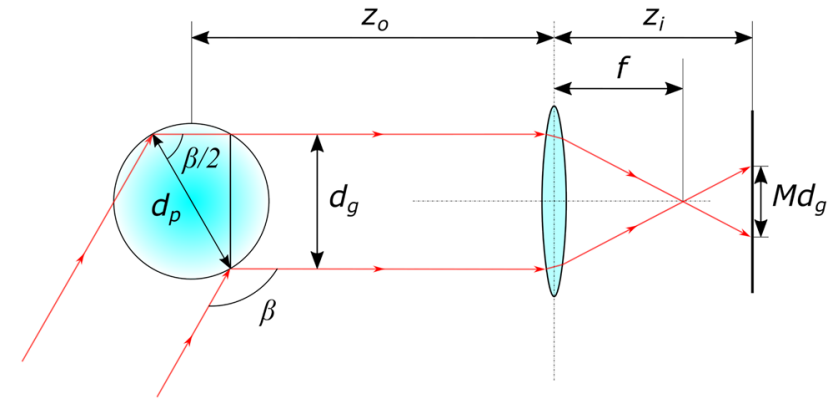

Fig. 26 Schematic representation of bubble glare points image formation. Only the main two light rays from the parallel laser illumination are considered that reflect directly towards the imager

externally and internally of the bubble (Fig. 26). The distance between them can be used to calculate the bubble diameter, while the midpoint yields the particle centre. Since the refractive index of helium and air are approximately the same, and the shift of the light ray direction within the soap film is negligible (the film thickness of a neutrally buoyant HFSB of $0.5 \mathrm{~mm}$ diameter is about $80 \mathrm{~nm}$, Eq. (33)), refraction can be neglected. Thus, the spherical bubble diameter can be obtained from geometric considerations:

$\mathrm{d}_{p}=\frac{\mathrm{d}_{g}}{\cos \left(\frac{\pi-\beta}{2}\right)}$,

where $\mathrm{d}_{g}$ is the distance between the bubble glare points and $\beta$ is the angle between the incoming light and the imaging direction. If the camera is positioned perpendicularly to the laser light sheet $\left(\beta=90^{\circ}\right)$, as in a typical planar PIV set-up, then eq. (A1.1) reduces to:

$\mathrm{d}_{p}=\sqrt{2} \mathrm{~d}_{g}$.

The size of a glare point $\delta_{g}$ can be obtained geometrically as a function of the bubble size and imaging conditions. Consider the case of a bubble being illuminated at a straight

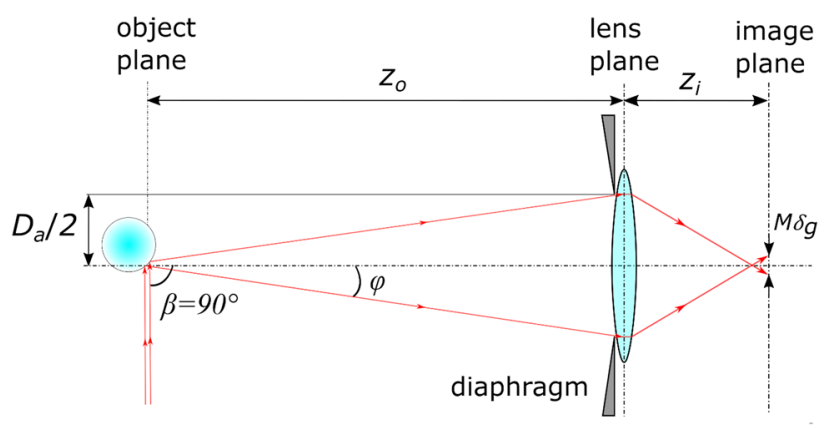

Fig. 27 Glare point size from light reflected externally on the bubble surface, imaged at $\beta=90^{\circ}$ relative to the illumination direction 


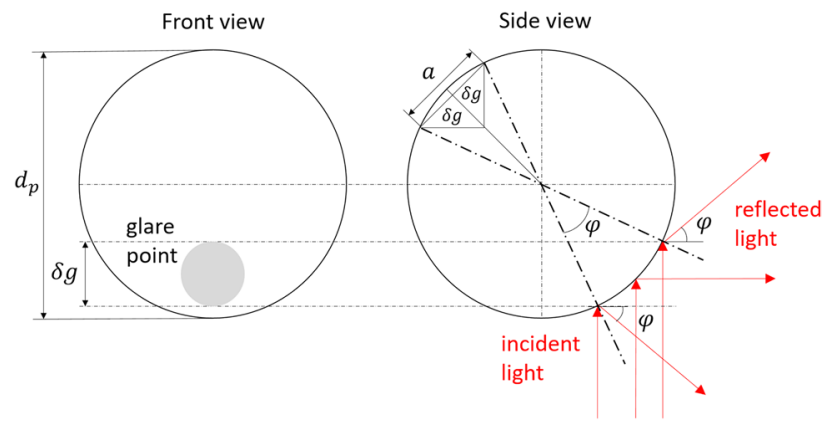

Fig. 28 Angle subtended by the illuminated surface area corresponding to the imaged glare point Finally, it is noted that if $\mathrm{d}_{g}<\mathrm{d}_{\text {diff }} / M$, then the glare points will merge, yielding extra uncertainty on the determination of the particle centre.

angle relative to the imaging direction ( $\beta=90^{\circ}$, Fig. 27). For simplicity, the centre of the glare point is positioned at the lens symmetry axis. The distance from the glare point to the lens is $z_{o}$. The angle $\varphi$ subtended by the illuminated area on the bubble surface (Fig. 28), corresponding to the glare point, is half the angle subtended by the aperture diameter $D_{a}$ (Fig. 27). For $z_{o} \gg D_{a} \gg d_{p}(\tan \varphi \approx \varphi)$, the angle $\varphi$ is given as:

$\varphi \approx \frac{\mathrm{D}_{a}}{2 z_{o}}$

The glare point size is obtained geometrically from Fig. 28 $(\sin \varphi / 2 \approx \varphi / 2)$ :

$\delta_{g}=\frac{a}{\sqrt{2}} \approx \frac{1}{2 \sqrt{2}} \mathrm{~d}_{p} \varphi$

Substituting eq. (A1.3) and $D_{a}=f / f_{\#}$ into eq. (A1.4) yields the glare point size in the object plane:

$\delta_{g} \approx \frac{1}{4 \sqrt{2}} \frac{\mathrm{d}_{p} f}{z_{0} f_{\#}}$.

Additionally, considering diffraction effects, the glare point size in the image plane is:

$\Delta_{g}=\sqrt{\left(M \delta_{g}\right)^{2}+\mathrm{d}_{\mathrm{diff}}^{2}}$

where $\mathrm{d}_{\text {diff }}$ is the diffraction limited diameter (Adrian and Yao 1985):

$\mathrm{d}_{\text {diff }}=2.44 f_{\#}(M+1) \lambda$.

In fact, diffraction effects become dominant. For instance, for $\mathrm{d}_{p}=0.5 \mathrm{~mm}, z_{o}=1.5 \mathrm{~m}, f=50 \mathrm{~mm}$ and $f_{\#}=16$ (experimental conditions in this paper), then $M \delta_{g}=6 \mathrm{~nm}$ and $\mathrm{d}_{\text {diff }}=21 \mu \mathrm{m}$. Therefore, $\Delta_{g} \approx \mathrm{d}_{\text {diff. }}$.

\section{Appendix 2}

\section{HFSB glare point intensity}

The light intensity from the light reflected on the external surface of the bubble (glare point 1 ) are only equal to that reflected on the internal part (glare point 2, see Fig. 26) when the observation angle with respect to the direction of the incoming laser light is $\beta=84^{\circ}$ (Dehaeck et al. 2005). At $90^{\circ}$ illumination, the authors showed that for an air bubbles in water, the intensity of the external glare point $I_{g 1}$ is about 5 times brighter than the internal one $I_{g 2}$. When the glare points are partially merged, the local maxima is, therefore, found at the centre of the brightest glare point, instead of at the particle centre, influencing the accuracy of particle centre identification.

This was tested for HFSB using previously obtained experimental data (Faleiros et al. 2018), where the glare points are sufficiently apart $\left(\mathrm{d}_{g} \sim 10 \mathrm{px}\right)$. Based on the particle images of approximately 2000 bubbles, it is found that the externally reflected glare points are only brighter in $70 \%$ of the recorded images (Fig. 29). The mean and standard deviation of the peak ratio $I_{g 1} / I_{g 2}$ are 1.34 and 0.67 , respectively.

In addition, the bubbles have been tracked in time to analyse the stability of the peak ratio within two time steps (Fig. 19). In $37.8 \%$ of the cases, the brightest glare point in frame 1 differed from that of frame 2, i.e. $I_{g 1} / I_{g 2}-1$ switched sign between the two frames, increasing the uncertainty on the particle centre identification from 0.1 px to about $\mathrm{Md}_{g}$.

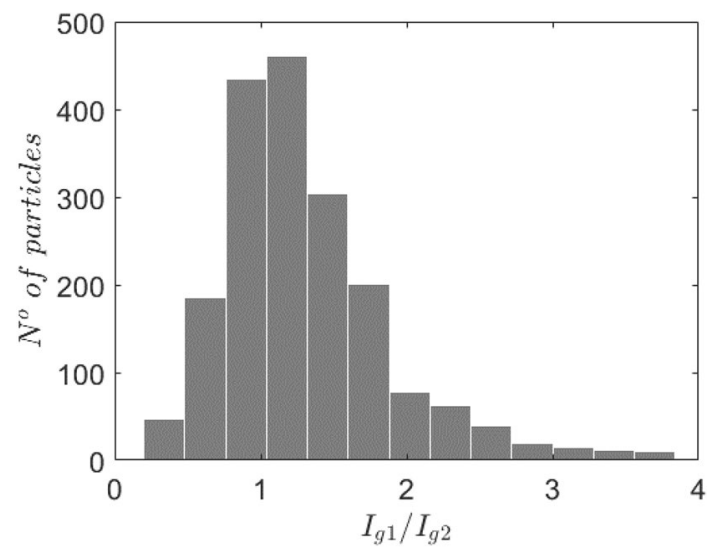

Fig. 29 Ratio of the bubble glare points intensity 
Acknowledgements This research project is part of the NEXT generation civil Tilt Rotor Interactional aerodynamic tail oPtimisation (NEXTTRIP) and the authors appreciate all the support received from its partners: NLR, DNW, DLR, Hit09 and Fokker.

Open Access This article is licensed under a Creative Commons Attribution 4.0 International License, which permits use, sharing, adaptation, distribution and reproduction in any medium or format, as long as you give appropriate credit to the original author(s) and the source, provide a link to the Creative Commons licence, and indicate if changes were made. The images or other third party material in this article are included in the article's Creative Commons licence, unless indicated otherwise in a credit line to the material. If material is not included in the article's Creative Commons licence and your intended use is not permitted by statutory regulation or exceeds the permitted use, you will need to obtain permission directly from the copyright holder. To view a copy of this licence, visit http://creativecommons.org/licenses/by/4.0/.

\section{References}

Adrian RJ, Yao CS (1985) Pulsed laser technique application to liquid and gaseous flows and the scattering power of seed materials. Appl Optics 24:44-52

Agüera N, Cafiero G, Astarita T, Discetti S (2016) Ensemble 3D PTV for high resolution turbulent statistics. Meas Sci Technol 27:124011

Basset AB (1888a) A treatise on hydrodynamics. Deighton Bell, London

Basset AB (1888b) On the Motion of a sphere in a Viscous Liquid. Philos T R Soc 179:43-63

Benedict LH, Gould RD (1996) Towards better uncertainty estimates for turbulence statistics. Exp Fluids 22:129-136

Benton SI, Visbal MR (2018) Understanding abrupt leading edge separation as a mechanism for the onset of dynamic stall. In: 2018 AIAA aerospace sciences meeting. p. 0356.

Bosbach J, Kühn M, Wagner C (2009) Large scale particle image velocimetry with helium filled soap bubbles. Exp Fluids 46:539-547

Bourgoin M, Qureshi NM, Baudet C, Cartellier A, Gagne Y (2011) Turbulent transport of finite sized material particles. J Phys Conf Ser 318:012005

Boussinesq (1885) Sur la résistance qu'oppose un fluide indéfini au repos. C R Acad Sci III 100:935

Boussinesq (1885) Applications â l'étude des potentiels (reedition 1969). Blanchard, Paris

Calzavarini E, Volk R, Bourgoin M, Lévêque E, Pinton J-F, Toschi F (2009) Acceleration statistics of finite-sized particles in turbulent flow: the role of Faxén forces. J Fluid Mech 630:179-189

Caridi GCA (2018) Development and application of helium-filled soap bubbles for large-scale PIV experiments in aerodynamics. PhD Thesis, Delft University of Technology.

Caridi GCA, Ragni D, Sciacchitano A, Scarano F (2016) HFSB seeding for large scale tomographic PIV in wind tunnels. Exp Fluids 57:190-202

Clift R, Grace J, Webe M (1978) Bubbles, drops and particles. Academic Press, New York

Dehaeck S, van Beeck JPAJ, Riethmuller ML (2005) Extended glare point velocimetry and sizing for bubbly flows. Exp Fluids 39:407

Drela M (1989) XFOIL: an analysis and design system for low Reynolds number airfoils. In: Mueller J (ed) Low reynolds number aerodynamics, Lecture notes Eng 54. Springer, New York.

Faleiros DE (2021) Soap bubbles for large-scale PIV: Generation, control and tracing accuracy. PhD Thesis, Delft University of Technology.
Faleiros DE, Tuinstra M, Sciacchitano A, Scarano F (2018) Helium-filled soap bubbles tracing fidelity in wall-bounded turbulence. Exp Fluids 59:56-68

Faleiros DE, Tuinstra M, Sciacchitano A, Scarano F (2019) Generation and control of helium-filled soap bubbles for PIV. Exp Fluids 60:40-56

Faxén (1922) Der Widerstand gegen die Bewegung einer starren Kugel in einer zähen Flüssigkeit, die zwischen zwei parallelen Ebenen Wänden eingeschlossen ist. Ann Phys (berl) 4:89-119

Gibeau B, Ghaemi S (2018) A modular 3D-printed helium-filled soap bubble generator for large-scale volumetric flow measurements. Exp Fluids 59:178-188

Gibeau B, Gingras D, Ghaemi S (2020) Evaluation of a full-scale heliumfilled soap bubble generator. Exp Fluids 61:28-45

Hale R W, Tan P, Ordway DE (1969) Experimental Investigation of Several Neutrally-Buoyant Bubble Generators for Aerodynamic Flow Visualization. Sage Action, Inc, SAI-RR 6901, AD-717 390.

Hale RW, Tan P, Stowell RC, Ordway DE (1971) Development of an integrated system for flow visualization in air using neutrally buoyant bubbles. Sage Action, Inc, SAI-RR 7107, AD-756 691.

Huhn F, Schanz D, Gesemann S, Dierksheide U, van de Meerendonk R, Schröder A (2017) Large-scale volumetric flow measurement in a pure thermal plume by dense tracking of helium-filled soap bubbles. Exp Fluids 58:116-134

Kerho MF, Bragg MB (1994) Neutrally buoyant bubbles used as flow tracers in air. Exp Fluids 16:393-400

Lazar E, Deblauw B, Glumac N, Dutton C, Elliott G (2010) A practical approach to PIV uncertainty analysis, In: 27th AIAA aerodynamic measurement technology and ground testing conference. p. 4355.

Lima Pereira LT, Ragni D, Avallone F, Scarano F (2020) Pressure fluctuations from large-scale PIV over a serrated trailing edge. Exp Fluids 61:71-87

Loth E (2008) Quasi-steady shape and drag of deformable bubbles and drops. Int J Multiph Flow 34:523-546

Loth E, Dorgan AJ (2009) An equation of motion for particles of finite Reynolds number and size. Environ Fluid Mech 9:187-206

Magnaudet J (1997) The forces acting on bubbles and rigid particles. In: ASME fluids engineering division summer meeting, FEDSM, pp. 22-26.

Magnaudet J, Eames I (2000) The motion of high-Reynolds-number bubbles in inhomogeneous flows. Annu Rev Fluid Mech 32:659-708

Maxey MR, Riley JJ (1983) Equation of motion for a small rigid sphere in a nonuniform flow. Phys Fluids 26:883-889

Mei R (1994) Flow due to an oscillating sphere and an expression for unsteady drag on the sphere at finite Reynolds number. J Fluid Mech 270:133-174

Mei R (1996) Velocity fidelity of flow tracer particles. Exp Fluids 22:1

Melling A (1997) Tracer particles and seeding for particle image velocimetry. Meas Sci Technol 8:1406

Michaelides EE (1997) The transient equation of motion for particles, bubbles, and droplets. J Fluids Eng 119:223-247

Morias KLL, Caridi GCA, Sciacchitano A, Scarano F (2016) Statistical characterization of helium-filled soap bubbles tracing fidelity for PIV. In: 18th international symposium on the application of laser and imaging techniques to fluid mechanics, Lisbon.

Müller D, Müller B, Renz U (2001) Three-dimensional particle-streak tracking (PST) velocity measurements of a heat exchanger inlet flow: A new method to measure all three air-flow velocity components in a plane is applied to a steady-state three-dimensional flow. Exp Fluids 30:645-656

Okuno Y, Fukuda T, Miwate Y, Kobayashi T (1993) Development of three dimensional air flow measuring method using soap bubbles. JSAE Rev 14(4):50-55

Qureshi NM, Arrieta U, Baudet C, Cartellier A, Gagne Y, Bourgoin M (2008) Acceleration statistics of inertial particles in turbulent flow. Eur Phys J B 66:531-536 
Raffel M, Willert CE, Scarano F, Kähler C, Wereley ST, Kompenhans J (2018) Particle image velocimetry-a practical guide, 3rd edn. Springer, Berlin

Ragni D, Schrijer F, van Oudheusden BW, Scarano F (2010) Particle tracer response across shocks measured by PIV. Exp Fluids 50:53-64

Reinders W (1994) Data report of the effect of in-flight icing on the 2D high lift model 5-6 in the low-speed wind tunnel LST. Internal report (Royal Netherlands Aerospace Centre) NLR-CR-1994-551.

Samimy M, Lele SK (1991) Motion of particles with inertia in a compressible free shear layer. Phys Fluids A 3:1915-1923

Scarano F (2013) Tomographic PIV: principles and practice. Meas Sci Technol 24:012001

Scarano F, van Oudheusden BW (2003) Planar velocity measurements of a two-dimensional compressible wake. Exp Fluids 34:430-441

Scarano F, Ghaemi S, Caridi GCA, Bosbach J, Dierksheide U, Sciacchitano A (2015) On the use of helium filled soap bubbles for large scale tomographic PIV in wind tunnel experiments. Exp Fluids $56: 42-53$

Schrijer FFJ, Scarano F, van Oudheusden BW (2006) Application of PIV in a Mach 7 double-ramp flow. Exp Fluids 41:353-363

Sciacchitano A, Wieneke B (2016) PIV uncertainty propagation. Measur. Sci Technol 27(8):084006

Sciacchitano A, Giaquinta D, Schneiders JFG, Scarano F, van Rooijen BD, Funes D E (2018) Quantitative flow visualization of a turboprop aircraft by robotic volumetric velocimetry. In: 18th international symposium on flow visualization, ETH Zurich.

Shi P, Rzehak R (2020) Lift forces on solid spherical particles in wallbounded flows. Chem Eng Sci 211:115264

Spoelstra A, de Martino NL, Terra W, Sciacchitano A, Scarano F (2019) On-site cycling drag analysis with the Ring of Fire. Exp Fluids 60:90-105

Terra W, Sciacchitano A, Shah YH (2019) Aerodynamic drag determination of a full-scale cyclist mannequin from large-scale PTV measurements. Exp Fluids 60:29-39

Usherwood JR, Cheney J A, Song J, Windsor SP, Stevenson JPJ, Dierksheide U, Nila A, Bomphrey RJ (2020) High aerodynamic lift from the tail reduces drag in gliding raptors. J Exp Biol 223:jeb214809

$\mathrm{Xu} \mathrm{H}$, Bodenschatz E (2008) Motion of inertial particles with size larger than Kolmogorov scale in turbulent flows. Phys D 237(14-17):2095-2100

Zeng L, Najjar F, Balachandar S, Fischer P (2009) Forces on a finite-sized particle located close to a wall in a linear shear flow. Phys Fluids 21:033302

Zhang B, Zerubia J, Olivo-Marin J-C (2007) Gaussian approximations of fluorescence microscope point-spread function models. App Optics 46:1819

Publisher's Note Springer Nature remains neutral with regard to jurisdictional claims in published maps and institutional affiliations. 